DRAFT VERSION SEPTEMBER 17, 2018

Typeset using LTTE $_{\mathrm{E}} \mathrm{X}$ twocolumn style in AASTeX61

\title{
OUTFLOWING $\mathrm{OH}^{+}$IN MARKARIAN 231: THE IONIZATION RATE OF THE MOLECULAR GAS
}

\author{
E. GonzÁlez-Alfonso, ${ }^{1,2}$ J. Fischer, ${ }^{3, *}$ S. Bruderer, ${ }^{4}$ M. L. N. Ashby, ${ }^{2}$ H. A. Smith, ${ }^{2}$ S. Veilleux, ${ }^{5}$ H. S. P. MÜller,${ }^{6}$ \\ K. P. STEWART, ${ }^{3}$ AND E. STURM ${ }^{4}$
}

\footnotetext{
${ }^{1}$ Universidad de Alcalá, Departamento de Física y Matemáticas, Campus Universitario, E-28871 Alcalá de Henares, Madrid, Spain

${ }^{2}$ Harvard-Smithsonian Center for Astrophysics, 60 Garden Street, Cambridge, MA 02138, USA

${ }^{3}$ Naval Research Laboratory, Remote Sensing Division, 4555 Overlook Ave SW, Washington, DC 20375, USA

${ }^{4}$ Max-Planck-Institute for Extraterrestrial Physics (MPE), Giessenbachstraße 1, 85748 Garching, Germany

${ }^{5}$ Department of Astronomy and Joint Space-Science Institute, University of Maryland, College Park, MD 20742, USA

${ }^{6}$ I. Physikalisches Institut, Universität zu Köln, Zülpicher Str. 77, 50937 Köln, Germany
}

\section{ABSTRACT}

The oxygen-bearing molecular ions $\mathrm{OH}^{+}, \mathrm{H}_{2} \mathrm{O}^{+}$, and $\mathrm{H}_{3} \mathrm{O}^{+}$are key species that probe the ionization rate of (partially) molecular gas that is ionized by X-rays and cosmic rays permeating the interstellar medium. We report Herschel far-infrared and submillimeter spectroscopic observations of $\mathrm{OH}^{+}$in Mrk 231, showing both ground-state P-Cygni profiles, and excited line profiles with blueshifted absorption wings extending up to $\approx 1000 \mathrm{~km} \mathrm{~s}^{-1}$. In addition, $\mathrm{OH}^{+}$probes an excited component peaking at central velocities, likely arising from the torus probed by the $\mathrm{OH}$ centimeter-wave megamaser. Four lines of $\mathrm{H}_{2} \mathrm{O}^{+}$are also detected at systemic velocities, but $\mathrm{H}_{3} \mathrm{O}^{+}$is undetected. Based on our earlier $\mathrm{OH}$ studies, we estimate an abundance ratio of $\mathrm{OH} / \mathrm{OH}^{+} \sim 5-10$ for the outflowing components and $\approx 20$ for the torus, and an $\mathrm{OH}^{+}$abundance relative to $\mathrm{H}$ nuclei of $\gtrsim 10^{-7}$. We also find high $\mathrm{OH}^{+} / \mathrm{H}_{2} \mathrm{O}^{+}$and $\mathrm{OH}^{+} / \mathrm{H}_{3} \mathrm{O}^{+}$ratios, both are $\gtrsim 4$ in the torus and $\gtrsim 10-20$ in the outflowing gas components. Chemical models indicate that these high $\mathrm{OH}^{+}$abundances relative to $\mathrm{OH}, \mathrm{H}_{2} \mathrm{O}^{+}$, and $\mathrm{H}_{3} \mathrm{O}^{+}$are characteristic of gas with a high ionization rate per unit density, $\zeta / n_{\mathrm{H}} \sim(1-5) \times 10^{-17} \mathrm{~cm}^{3} \mathrm{~s}^{-1}$ and $\sim(1-2) \times 10^{-16} \mathrm{~cm}^{3} \mathrm{~s}^{-1}$ for the above components, respectively, an ionization rate of $\zeta \sim(0.5-2) \times 10^{-12} \mathrm{~s}^{-1}$, and a low molecular fraction, $f_{\mathrm{H}_{2}} \sim 0.25$. X-rays appear to be unable to explain the inferred ionization rate, and thus we suggest that low-energy $(10-400 \mathrm{MeV})$ cosmic-rays are primarily responsible for the ionization with $\dot{M}_{\mathrm{CR}} \sim 0.01 \mathrm{M}_{\odot} \mathrm{yr}^{-1}$ and $\dot{E}_{\mathrm{CR}} \sim 10^{44} \mathrm{erg} \mathrm{s}^{-1}$, the latter corresponding to $\sim 1 \%$ of the AGN luminosity and similar to the energetics of the molecular outflow. We suggest that cosmic-rays accelerated in the forward shock associated with the molecular outflow are responsible for the ionization, as they diffuse through the outflowing molecular phase downstream.

Keywords: Line: formation - Galaxies: ISM - ISM: jets and outflows - Infrared: galaxies - Submillimeter: galaxies

\footnotetext{
* Current address: George Mason University, Department of Physics \& Astronomy, MS 3F3, 4400 University Drive, Fairfax, VA 22030, USA
} 


\section{INTRODUCTION}

Massive and powerful molecular outflows in galaxies have been detected and reported in a number of species, including $\mathrm{OH}$ in the far-infrared (Fischer et al. 2010; Sturm et al. 2011; Spoon et al. 2013; Veilleux et al. 2013a; Stone et al. 2016; González-Alfonso et al. 2014, 2017, hereafter GA14 and $\mathrm{GA} 17$ ) and $\mathrm{CO}, \mathrm{HCN}, \mathrm{HCO}^{+}$, and $\mathrm{CS}$ at (sub)millimeter wavelengths (e.g. Sakamoto et al. 2009; Feruglio et al. 2010, 2015; Alatalo et al. 2011, 2015; Cicone et al. 2012, 2014; Aalto et al. 2012, 2015; García-Burillo et al. 2015; Lindberg et al. 2016; Veilleux et al. 2017). Each species gives specific information on the physical and chemical conditions of the outflowing molecular gas, as a result of the different excitation mechanisms, varying sensitivity to the physical conditions (radiation field, gas temperature, column and volume densities), and the diversity of chemical reations that produce and destroy the molecules. In particular, the chemistry of the molecular gas is strongly influenced by the extreme environments in galaxy nuclei where the gas is subject to strong shocks, hard radiation fields (X-rays, UV), and cosmic rays (CRs). These physical processes can be studied via the chemical pathways they cause, as the molecular abundances and observed line strengths are constrained by the primary effects that X-rays and CRs have on the thermal state, partial ionization, and dissociation of the exposed molecular gas.

The transient $\mathrm{OH}^{+}$, with its rotational transitions in the far-IR and submillimeter domains, is one of the most suitable molecular species to trace the ionization rate of partially molecular gas and to constrain the sources of that ionization. CRs and X-rays are able to penetrate large columns of gas ionizing $\mathrm{H}$ and $\mathrm{H}_{2}$ (e.g., Herbst \& Klemperer 1973; Maloney et al. 1996; Meijerink et al. 2011; Bayet et al. 2011) at a rate $\zeta$; a relatively simple chain of ion-neutral reactions then leads to the formation of $\mathrm{OH}^{+}$, which is rapidly destroyed either by reacting with $\mathrm{H}_{2}$ or by dissociative recombination, at a rate proportional to $n_{\mathrm{H}}$. Therefore, the equilibrium abundance of $\mathrm{OH}^{+}$is sensitive to both $\zeta / n_{\mathrm{H}}$ and the molecular fraction $f_{\mathrm{H} 2}$, constraining the fluxes of CRs and X-rays that permeate the molecular gas.

González-Alfonso et al. (2013) (hereafter GA13) reported on and analyzed Herschel/PACS (Pilbratt et al. 2010; Poglitsch et al. 2010) detections of excited O-bearing molecular ions $\left(\mathrm{OH}^{+}, \mathrm{H}_{2} \mathrm{O}^{+}, \mathrm{H}_{3} \mathrm{O}^{+}\right)$in both $\mathrm{NGC} 4418$ and Arp 220. The detection of excited lines of $\mathrm{OH}^{+}, \mathrm{H}_{2} \mathrm{O}^{+}$, and $\mathrm{H}_{3} \mathrm{O}^{+}$in both galaxies allowed us to derive estimates of the ionization rates per hydrogen nucleus density $\left(\zeta / n_{\mathrm{H}}\right)$, and to place a lower limit on the ionization rate $\zeta$ of several hundreds times the typical rate inferred in the Milky Way. In Arp 220, the ground-state $\mathrm{OH}^{+}$lines show P-Cygni profiles (Rangwala et al. 2011) indicative of outflowing gas, and appear to arise in the more spatially extended disk where the ionization rate is significantly lower than in the nucleus (van der Tak et al. 2016).

In the present study, we extend our work on O-bearing molecular ions to Mrk 231, the closest QSO, the most luminous ultraluminous infrared galaxy (ULIRG) in the local $(z<0.1)$ Universe, and the most extensively studied extragalactic molecular outflow (Fischer et al. 2010; Feruglio et al. 2010, 2015; Sturm et al. 2011; Cicone et al. 2012; Aalto et al. 2012, 2015; Lindberg et al. 2016, GA14, GA17). The Herschel/PACS observations of Mrk 231 show prominent absorption in the line wings of $\mathrm{OH}^{+}$up to velocities of $\approx 1000$ $\mathrm{km} \mathrm{s}^{-1}$, together with very excited absorption at systemic velocities. We analyze the observations of $\mathrm{OH}^{+}$in combination with those of $\mathrm{H}_{2} \mathrm{O}^{+}$and $\mathrm{H}_{3} \mathrm{O}^{+}$, in the framework of the $\mathrm{OH}$ observations and radiative tranfer models reported in GA17. We also include in the analysis the Herschel/SPIRE observations of the ground-state lines of $\mathrm{OH}^{+}$and $\mathrm{H}_{2} \mathrm{O}^{+}$, which show prominent emission features (van der Werf et al. 2010). The inferred molecular abundances are further interpreted in terms of chemical models, which enable us to constrain $\zeta / n_{\mathrm{H}}$ and $f_{\mathrm{H} 2}$ for both the quiescent and outflowing components, as well as the ionization rate $\zeta$ upon conservative assumptions on the gas density. We then derive the energetics associated with low-energy CRs, which are most likely responsible for the ionization, and link these CRs to the forward shock associated with the molecular outflow. The observations are described in $\$ 2$, the analysis is developed in $\$ 3$, and our main findings are discussed in $\$ 4$. As in GA17, we adopt a distance to Mrk 231 of $186 \mathrm{Mpc}$, assuming a flat Universe with $H_{0}=71 \mathrm{~km} \mathrm{~s}^{-1} \mathrm{Mpc}^{-1}$ and $\Omega_{\mathrm{M}}=0.27$, with $z=0.04218$.

\section{OBSERVATIONS}

Most PACS data presented here were observed as part of the Herschel Open Time (OT2) program to obtain a full highresolution far-infrared specrum of Mrk 231 (PI: J. Fischer); a few of the spectra presented were observed during the science demonstration observations of the SHINING program (PI: E. Sturm). As described in GA14, the spectra were observed in high spectral sampling range-mode using first and second orders of the grating. The velocity resolution of PACS in first order ranges from $\approx 320$ to $180 \mathrm{~km} \mathrm{~s}^{-1}$ over the wavelength range from 105 to $190 \mu \mathrm{m}$, and in second order from $\approx 210$ to $110 \mathrm{~km} \mathrm{~s}^{-1}$ from 52 to 98 microns. The data reduction was mostly done using the PACS reduction and calibration pipeline (ipipe) included in HIPE 14.0.1, with calibration tree version 72, using an oversampling of 4 and fully independent channels (an upsample parameter of 1). Both the molecular absorption lines and the continua are effectively point-like in Mrk 231, and we have thus used the point source calibrated spectra "c129", produced by scaling the emission from the central $\approx 9^{\prime \prime} \times 9^{\prime \prime}$ spatial pixel to the total emission from the central $3 \times 3$ spaxels ("c9"), which is itself scaled according 
to the point-source correction (see also GA17). The absolute flux scale is robust to potential pointing jitter, with continuum flux reproducibility of $\pm 15 \%$.

The Herschel/SPIRE spectrum of Mrk 231 was observed by the Open Time Key Project Hercules (PI: P. van der Werf). The detection of the $\mathrm{OH}^{+}$and $\mathrm{H}_{2} \mathrm{O}^{+}$ground-state lines was reported by van der Werf et al. (2010) using the observations carried out on OD 209 (ObsID: 1342187893), but here we use the more sensitive observation from the same project taken in OD 558 (Rosenberg et al. 2015). The SPIRE spectrometer observations cover the wavelength range 191-671 $\mu \mathrm{m}$ with two spatial arrays covering two bands: $\operatorname{SSW}(191-318 \mu \mathrm{m})$ and $\operatorname{SLW}(294-671 \mu \mathrm{m})$. The HIPE 15.0.1 apodized spectra were downloaded from the archive. Mrk 231 is a point source for SPIRE FTS (HPBW $\geq 17^{\prime \prime}$ ), and therefore only the central detector spectra were used. In HR mode, the unapodized spectral resolution is FWHM $\left(\mathrm{km} \mathrm{s}^{-1}\right)=1.4472 \lambda(\mu \mathrm{m})$, but we have used the apodized spectra resulting in a FWHM a factor of 1.5 higher $^{1}$. The spectroscopic parameters used for line identification and radiative transfer modeling were taken from the spectral line catalogs of the CDMS $\left(\mathrm{OH}^{+}\right.$and $\mathrm{H}_{2} \mathrm{O}^{+}$, Müller et al. 2001, 2005). and JPL $\left(\mathrm{H}_{3} \mathrm{O}^{+}\right.$, Pickett et al. 1998); improved parameters for $\mathrm{OH}^{+}$have been recently reported by Markus et al. (2016).

The observations of the O-bearing molecular cations used in this study are summarized in Table 1 and the equivalent widths and fluxes of the PACS and SPIRE lines are listed in Tables 2 and 3, respectively.

\subsection{The $\mathrm{OH}^{+}$spectra}

The $\mathrm{OH}^{+}$observations, from both PACS (panels a-g) and SPIRE (panel h) are displayed in Fig. 1, together with the adopted baselines (dashed lines). In all cases, we have fitted polynomials of degree 1 around the (expected) line position to minimize the uncertainties in the velocity extent of the line wings. The most uncertain baseline corresponds to panel d, owing to the proximity of the strong $\mathrm{H}_{2} \mathrm{O} 2_{20}-1_{11}$ absorption line and, at shorter wavelengths, the PACS $100 \mu \mathrm{m}$ gap.

\subsubsection{Detections and potential contaminations}

Potential contamination by lines of other molecular species is indicated in Fig. 1. In panel a, the $\mathrm{OH}^{+} 2_{1}-1_{1}$ line is truncated at blueshifted velocities due to the proximity of the [C II] $158 \mu \mathrm{m}$ line; we nevertheless use the $\mathrm{OH}^{+}$absorption at central velocities to compare with model predictions in $\S 3$. In panel $b$, the $\mathrm{OH}^{+} 2_{3}-1_{2}$ line is closely blended with the $\mathrm{NH} 2_{2}-1_{1}$ line at $\approx 153.1 \mu \mathrm{m}$, as the $\mathrm{NH} 2_{3}-1_{2}$ line at $\approx 153.35 \mu \mathrm{m}$ is clearly detected. Nevertheless, the overall profile is dominated by $\mathrm{OH}^{+}$as previously suggested
Table 1. Obs ID of the $\mathrm{OH}^{+}, \mathrm{H}_{2} \mathrm{O}^{+}$, and $\mathrm{H}_{3} \mathrm{O}^{+}$lines in Mrk 231 .

\begin{tabular}{|c|c|c|}
\hline Transition & $\begin{array}{c}\lambda_{\text {rest }} \\
(\mu \mathrm{m})\end{array}$ & Obs ID \\
\hline $\mathrm{OH}^{+} \quad 1_{0}-0_{1}$ & 329.761 & 1342210493 \\
\hline $\mathrm{OH}^{+} \quad 1_{2}-0_{1}$ & 308.488 & 1342210493 \\
\hline $\mathrm{OH}^{+} \quad 1_{1}-0_{1}$ & 290.193 & 1342210493 \\
\hline $\mathrm{OH}^{+} 2_{2}-1_{2}$ & 147.768 & 1342253535 \\
\hline $\mathrm{OH}^{+} \quad 2_{1}-1_{0}$ & 148.696 & 1342253535 \\
\hline $\mathrm{OH}^{+} 2_{2}-1_{1}$ & 152.369 & 1342253536 \\
\hline $\mathrm{OH}^{+} \quad 2_{3}-1_{2}$ & 152.989 & 1342253536 \\
\hline $\mathrm{OH}^{+} 2_{1}-1_{1}$ & 158.437 & 1342186811 \\
\hline $\mathrm{OH}^{+} \quad 3_{2}-2_{1}$ & 101.265 & 1342253530 \\
\hline $\mathrm{OH}^{+} \quad 3_{3}-2_{2}$ & 101.698 & 1342253530 \\
\hline $\mathrm{OH}^{+} \quad 3_{4}-2_{3}$ & 101.921 & 1342253530 \\
\hline $\mathrm{OH}^{+} \quad 3_{2}-2_{2}$ & 103.909 & 1342253530 \\
\hline $\mathrm{OH}^{+} \quad 4_{3}-3_{2}$ & 76.245 & 1342253536 \\
\hline $\mathrm{OH}^{+} \quad 4_{4}-3_{3}$ & 76.399 & 1342253536 \\
\hline $\mathrm{OH}^{+} \quad 4_{5}-3_{4}$ & 76.510 & 1342253536 \\
\hline $\mathrm{OH}^{+} 5_{4}-4_{3}$ & 61.168 & 1342253532 \\
\hline $\mathrm{OH}^{+} 5_{5}-4_{4}$ & 61.248 & 1342253532 \\
\hline $\mathrm{OH}^{+} \quad 5_{6}-4_{5}$ & 61.315 & 1342253532 \\
\hline $\mathrm{oH}_{2} \mathrm{O}^{+} 1_{11} \frac{1}{2}-0_{00} \frac{1}{2}$ & 263.077 & 1342210493 \\
\hline $\mathrm{oH}_{2} \mathrm{O}^{+} 1_{11} \frac{3}{2}-0_{00} \frac{1}{2}$ & 268.850 & 1342210493 \\
\hline $\mathrm{pH}_{2} \mathrm{O}^{+} 2_{21} \frac{3}{2}-1_{10} \frac{1}{2}$ & 104.719 & 1342253530 \\
\hline $\mathrm{pH}_{2} \mathrm{O}^{+} 2_{21} \frac{5}{2}-1_{10} \frac{3}{2}$ & 105.742 & 1342253530 \\
\hline $\mathrm{oH}_{2} \mathrm{O}^{+} 3_{22} \frac{5}{2}-211 \frac{3}{2}$ & 88.978 & 1342253539 \\
\hline $\mathrm{oH}_{2} \mathrm{O}^{+} 3_{22} \frac{7}{2}-211 \frac{5}{2}$ & 89.590 & 1342253539 \\
\hline $\mathrm{oH}_{2} \mathrm{O}^{+} 3_{13} \frac{5}{2}-202 \frac{3}{2}$ & 143.265 & 1342253534 \\
\hline $\mathrm{oH}_{2} \mathrm{O}^{+} 3_{13} \frac{7}{2}-202 \frac{5}{2}$ & 143.810 & 1342253534 \\
\hline $\mathrm{oH}_{2} \mathrm{O}^{+} 4_{04} \frac{9}{2}-3_{13} \frac{7}{2}$ & 145.917 & 1342253535 \\
\hline $\mathrm{oH}_{2} \mathrm{O}^{+} 4_{04} \frac{7}{2}-313 \frac{5}{2}$ & 146.216 & 1342253535 \\
\hline $\mathrm{oH}_{3} \mathrm{O}^{+} 4_{3}^{-}-3_{3}^{+}$ & 69.538 & 1342253534 \\
\hline $\mathrm{pH}_{3} \mathrm{O}^{+} 4_{1}^{-}-3_{1}^{+}$ & 70.684 & 1342253534 \\
\hline $\mathrm{oH}_{3} \mathrm{O}^{+} 4_{0}^{-}-3_{0}^{+}$ & 70.827 & 1342253534 \\
\hline $\mathrm{pH}_{3} \mathrm{O}^{+} 3_{2}^{-}-2_{2}^{+}$ & 82.274 & 1342253537 \\
\hline $\mathrm{pH}_{3} \mathrm{O}^{+} 3_{1}^{-}-2_{1}^{+}$ & 82.868 & 1342253537 \\
\hline
\end{tabular}



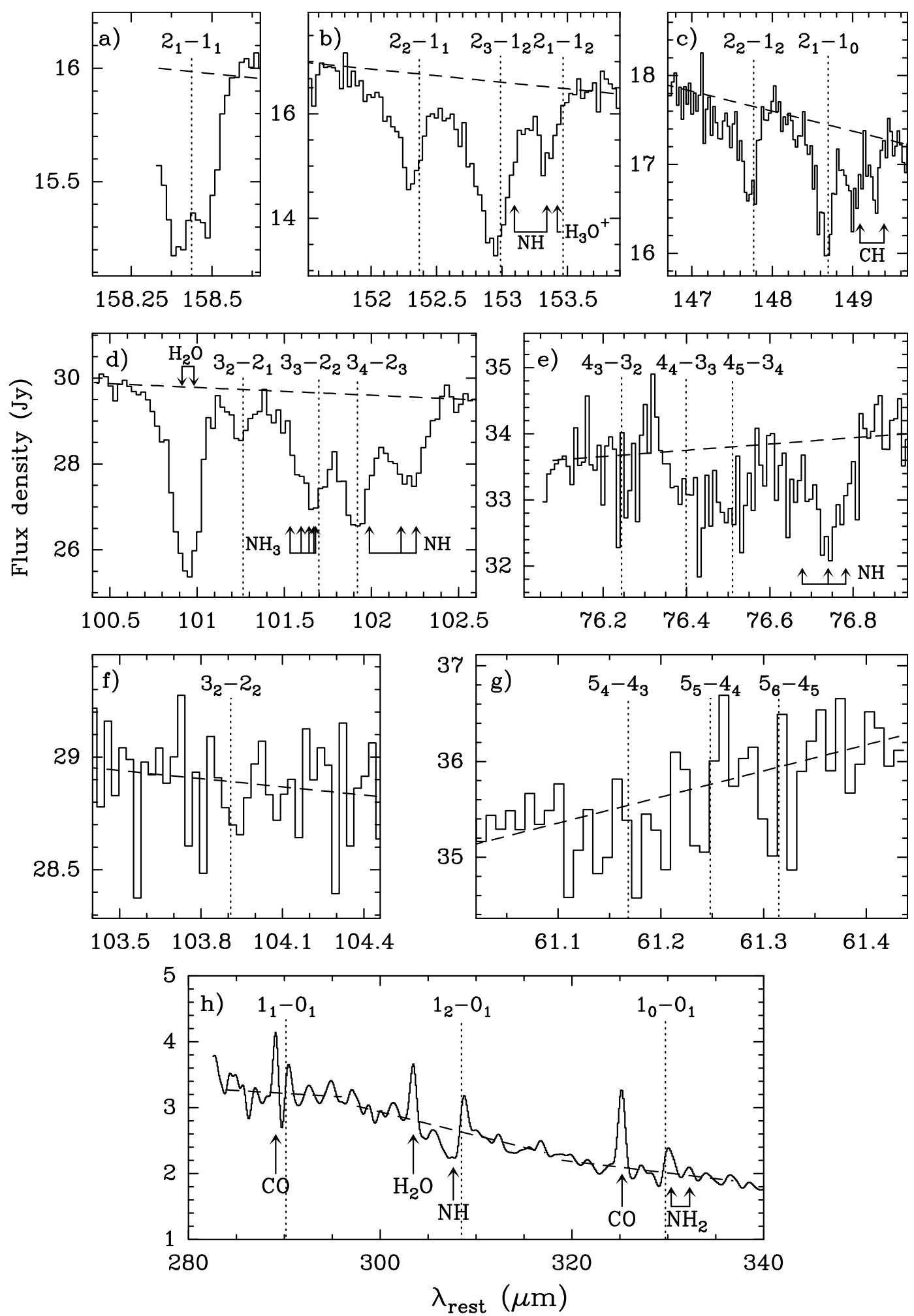

Figure 1. The observed spectra around the $\mathrm{OH}^{+}$lines in Mrk 231, with the adopted baselines (dashed lines). The rest wavelengths, marked by vertical dotted lines, are calculated with respect to the systemic redshift of $z=0.04218$. Contributions to the spectra by $\mathrm{NH}$ (panels $\mathrm{b}, \mathrm{d}$, e and h), $\mathrm{NH}_{2}$ (panel h), $\mathrm{NH}_{3}$ (panel d), $\mathrm{CH}$ (panel c), and $\mathrm{CO}$ (panel h) are also indicated. In panel a, the $\mathrm{OH}^{+} 2_{1}-1_{1}$ line is truncated at blueshifted velocities due to the proximity of the [C II] $158 \mu \mathrm{m}$ line. 
Table 2. Line equivalent widths and fluxes of the PACS lines

\begin{tabular}{|c|c|c|c|}
\hline Transition & $\begin{array}{c}\text { Velocities }^{\mathrm{a}} \\
\left(\mathrm{km} \mathrm{s}^{-1}\right)\end{array}$ & $\begin{array}{c}W_{\mathrm{eq}}^{\mathrm{b}, \mathrm{c}} \\
\left(\mathrm{km} \mathrm{s}^{-1}\right)\end{array}$ & $\begin{array}{c}\text { Flux }^{\mathrm{c}} \\
\left(\mathrm{Jy} \mathrm{km} \mathrm{s}^{-1}\right)\end{array}$ \\
\hline \multirow[t]{2}{*}{$\mathrm{OH}^{+} \quad 2_{2}-1_{2}$} & {$[-300,200]$} & $22.2(2.8)$ & $-394(49)$ \\
\hline & {$[-1000,-300]$} & $13.4(3.0)$ & $-237(54)$ \\
\hline \multirow[t]{2}{*}{$\mathrm{OH}^{+} \quad 2_{1}-1_{0}$} & {$[-300,200]$} & $31.5(2.6)$ & $-553(45)$ \\
\hline & {$[-1000,-300]$} & $10.4(3.0)$ & $-182(53)$ \\
\hline \multirow[t]{2}{*}{$\mathrm{OH}^{+} \quad 2_{2}-1_{1}$} & {$[-300,200]$} & $46.5(3.0)$ & $-774(50)$ \\
\hline & {$[-1000,-300]$} & $26.5(3.7)$ & $-441(60)$ \\
\hline \multirow[t]{2}{*}{$\mathrm{OH}^{+} \quad 2_{3}-1_{2}$} & {$[-300,200]$} & $80.0(3.2)^{\mathrm{d}}$ & $-1320(52)^{\mathrm{d}}$ \\
\hline & {$[-1000,-300]$} & $49.2(3.6)$ & $-812(60)$ \\
\hline $\mathrm{OH}^{+} \quad 2_{1}-1_{1}$ & {$[-300,200]$} & $14.3(0.6)^{\mathrm{d}}$ & $-229(9)^{\mathrm{d}}$ \\
\hline $\mathrm{OH}^{+} \quad 3_{2}-2_{1}$ & {$[-300,200]$} & $14.1(1.3)$ & $-419(39)$ \\
\hline \multirow[t]{2}{*}{$\mathrm{OH}^{+} \quad 3_{3}-2_{2}$} & {$[-300,200]$} & $38.4(1.3)^{\mathrm{d}}$ & $-1143(38)^{\mathrm{d}}$ \\
\hline & {$[-1000,-300]$} & $20.6(1.5)^{\mathrm{d}}$ & $-615(45)^{d}$ \\
\hline $\mathrm{OH}^{+} \quad 3_{4}-2_{3}$ & {$[-300,200]$} & $44.8(1.2)^{\mathrm{d}}$ & $-1334(37)^{\mathrm{d}}$ \\
\hline $\mathrm{OH}^{+} \quad 4_{3}-3_{2}$ & {$[-300,200]$} & $<3.7$ & $>-124$ \\
\hline $\mathrm{OH}^{+} 4_{4}-3_{3}$ & {$[-300,200]$} & $7.6(2.3)$ & $-255(76)$ \\
\hline $\mathrm{OH}^{+} 4_{5}-3_{4}$ & {$[-300,200]$} & $13.0(2.1)$ & $-440(69)$ \\
\hline $\mathrm{OH}^{+} \quad 3_{2}-2_{2}$ & {$[-300,200]$} & $<3.0$ & $>-86$ \\
\hline $\mathrm{OH}^{+} \quad 5_{4}-4_{3}$ & {$[-300,200]$} & $<4.4$ & $>-158$ \\
\hline $\mathrm{OH}^{+} \quad 5_{5}-4_{4}$ & {$[-300,200]$} & $<4.8$ & $>-172$ \\
\hline $\mathrm{OH}^{+} 5_{6}-4_{5}$ & {$[-300,200]$} & $<4.7$ & $>-168$ \\
\hline $\mathrm{pH}_{2} \mathrm{O}^{+} 2_{21} \frac{3}{2}-1_{10} \frac{1}{2}$ & {$[-300,200]$} & $<4.6^{\mathrm{d}}$ & $>-134^{\mathrm{d}}$ \\
\hline $\mathrm{pH}_{2} \mathrm{O}^{+} 2_{21} \frac{5}{2}-1_{10} \frac{3}{2}$ & {$[-300,200]$} & $4.3(1.1)$ & $-125(32)$ \\
\hline $\mathrm{oH}_{2} \mathrm{O}^{+} 3_{22} \frac{5}{2}-211 \frac{3}{2}$ & {$[-300,200]$} & $<3.8$ & $>-120$ \\
\hline $\mathrm{oH}_{2} \mathrm{O}^{+} 3_{22} \frac{7}{2}-211 \frac{5}{2}$ & {$[-300,200]$} & $<3.9$ & $>-120$ \\
\hline $\mathrm{oH}_{2} \mathrm{O}^{+} 3_{13} \frac{5}{2}-202 \frac{3}{2}$ & {$[-300,200]$} & $8.2(2.0)$ & $-150(36)$ \\
\hline $\mathrm{oH}_{2} \mathrm{O}^{+} 3_{13} \frac{7}{2}-202 \frac{5}{2}$ & {$[-300,200]$} & $8.4(1.6)$ & $-154(29)$ \\
\hline $\mathrm{oH}_{2} \mathrm{O}^{+} 4_{04} \frac{9}{2}-313 \frac{7}{2}$ & {$[-300,200]$} & $<3.8$ & $>-58$ \\
\hline $\mathrm{oH}_{2} \mathrm{O}^{+} 4_{04} \frac{7}{2}-313 \frac{5}{2}$ & {$[-300,200]$} & $<3.2$ & $>-48$ \\
\hline $\mathrm{oH}_{3} \mathrm{O}^{+} 4_{3}^{-}-3_{3}^{+}$ & {$[-300,200]$} & $<2.7$ & $>-92$ \\
\hline $\mathrm{pH}_{3} \mathrm{O}^{+} 4_{1}^{-}-3_{1}^{+}$ & {$[-300,200]$} & $<3.1$ & $>-106$ \\
\hline $\mathrm{oH}_{3} \mathrm{O}^{+} 4_{0}^{-}-3_{0}^{+}$ & {$[-300,200]$} & $<2.5$ & $>-86$ \\
\hline $\mathrm{pH}_{3} \mathrm{O}^{+} 3_{2}^{-}-2_{2}^{+}$ & {$[-300,200]$} & $<4.2$ & $>-138$ \\
\hline $\mathrm{pH}_{3} \mathrm{O}^{+} 3_{1}^{-}-2_{1}^{+}$ & {$[-300,200]$} & $<3.7$ & $>-120$ \\
\hline
\end{tabular}

a Velocity range in which equivalent widths and fluxes are calculated

b Equivalent width

${ }^{c}$ Numbers in parenthesis are $1 \sigma$ uncertainties, and upper or lower limits correspond to $2 \sigma$.

${ }^{d}$ Partially contaminated by lines of other species (see text)
Table 3. Line equivalent widths and fluxes of the SPIRE lines

\begin{tabular}{|c|c|c|c|}
\hline Transition & $\begin{array}{c}\text { Velocities }^{\mathrm{a}} \\
\left(\mathrm{km} \mathrm{s}^{-1}\right)\end{array}$ & $\begin{array}{c}W_{\mathrm{eq}}^{\mathrm{b}, \mathrm{c}} \\
\left(\mathrm{km} \mathrm{s}^{-1}\right)\end{array}$ & $\begin{array}{c}\text { Flux }^{\mathrm{c}} \\
\left(\mathrm{Jy} \mathrm{km} \mathrm{s}^{-1}\right)\end{array}$ \\
\hline \multirow[t]{2}{*}{$\mathrm{OH}^{+} \quad 1_{0}-0_{1}$} & {$[-100,850]$} & $-146(18)^{\mathrm{d}}$ & $293(36)^{d}$ \\
\hline & {$[-1200,-300]$} & $65(18)$ & $-130(36)$ \\
\hline \multirow[t]{2}{*}{$\mathrm{OH}^{+} \quad 1_{2}-0_{1}$} & {$[-100,850]$} & $-145(15)$ & $381(39)$ \\
\hline & {$[-1200,-200]$} & $135(15)^{d}$ & $-354(39)^{\mathrm{d}}$ \\
\hline \multirow[t]{2}{*}{$\mathrm{OH}^{+} \quad 1_{1}-0_{1}$} & {$[-100,850]$} & $-79(27)$ & $253(88)$ \\
\hline & {$[-730,-90]$} & $67(27)^{\mathrm{d}}$ & $-215(88)^{\mathrm{d}}$ \\
\hline $\mathrm{oH}_{2} \mathrm{O}^{+} 1_{11} \frac{1}{2}-0_{00} \frac{1}{2}$ & {$[-400,400]$} & $-65(10)^{\mathrm{d}}$ & $268(42)^{\mathrm{d}}$ \\
\hline
\end{tabular}

a Velocity range in which equivalent widths and fluxes are calculated

b Equivalent width

${ }^{c}$ Numbers in parenthesis are $1 \sigma$ uncertainties

${ }^{\mathrm{d}}$ Contaminated by lines of other species (see text)

based on ISO observations (González-Alfonso et al. 2008). We also indicate in panel $\mathrm{b}$ the position of the very highlying $\mathrm{H}_{3} \mathrm{O}^{+} 12^{-}-12^{+}$transition $\left(E_{\text {lower }} \approx 1400 \mathrm{~K}\right)$, detected in Arp 220 (GA13) but absent in Mrk 231; the nearby (intrinsically weak) $\mathrm{OH}^{+} 2_{1}-1_{2}$ line is also not detected. In panel $\mathrm{c}$, we detect (blueshifted) absorption in the $\mathrm{CH}$ ground-state doublet at $149 \mu \mathrm{m}$, but it is resolved from the neighboring $\mathrm{OH}^{+} 2_{1}-1_{0}$ line.

The redshift of Mrk 231 places the $\mathrm{OH}^{+} 3_{J}-2_{J^{\prime}}$ lines $\left(E_{\text {lower }} \approx 140 \mathrm{~K}, \lambda_{\text {rest }} \sim 102 \mu \mathrm{m}\right)$ redward $\left(\lambda_{\text {obs }} \sim 106 \mu \mathrm{m}\right)$ of the $100 \mu \mathrm{m}$ range short wavelength leakage region of PACS, allowing its observation (Fig. 1d) ${ }^{2}$. Since NH has a spectrum similar to $\mathrm{OH}^{+}$, contamination is also found in the $\mathrm{OH}^{+}$ $3_{4}-2_{3}$ line at $101.9 \mu \mathrm{m}$ by the $\mathrm{NH} 3_{2}-2_{1}$ line at $102.0 \mu \mathrm{m}$, as the blend of the $\mathrm{NH} 3_{3}-2_{2}$ and $3_{4}-2_{3}$ lines at $102.2 \mu \mathrm{m}$ is well detected (panel d). Nevertheless, the $3_{3}-2_{2}$ line of $\mathrm{OH}^{+}$at $101.7 \mu \mathrm{m}$ is strongly detected with evidence for blueshifted absorption, though contamination by the $\mathrm{NH}_{3}$ $5_{K}-4_{K}$ lines (with $K=0, \ldots, 4$ ) should be considered (see $\S 3)$. The strength of the intrinsically weak $\mathrm{OH}^{+} 3_{2}-2_{1}$ line at $101.3 \mu \mathrm{m}$ is doubtful owing to the proximity of the $\mathrm{H}_{2} \mathrm{O}$ $101 \mu \mathrm{m}$ line and the uncertain continuum level. In panel $\mathrm{e}$, the $\mathrm{OH}^{+} 4_{5}-3_{4}$ and $4_{4}-3_{3}$ lines are detected at systemic velocities, with hints of absorption in the $4_{3}-3_{2}$ component; the $\mathrm{OH}^{+} 4_{J}-3_{J-1}$ lines are well separated from the corresponding $\mathrm{NH}$ lines. In panels $\mathrm{f}$ and $\mathrm{g}$, the spectra around the

\footnotetext{
${ }^{2}$ In NGC 4418 and Arp 220, these lines fell within the $100 \mu \mathrm{m}$ gap and were thus not observed (GA13).
} 
undetected $\mathrm{OH}^{+} 3_{2}-2_{2}$ and $5_{J}-4_{J-1}$ lines are shown, and are used in $\$ 3$ to check for overall consistency with the models. Panel h shows the SPIRE spectrum around the $\mathrm{OH}^{+}$groundstate $1_{J}-0_{1}$ lines. The $1_{1}-0_{1}$ line is contaminated on its blue side by the CO $9-8$ line at $289.12 \mu \mathrm{m}$, the $1_{2}-0_{1}$ line has potential contamination by the ground-state $\mathrm{NH} 1_{2}-0_{1}$ line at $\approx 307.6 \mu \mathrm{m}$, and the emission component of the $1_{0}-0_{1}$ line is most likely contaminated by $\mathrm{NH}_{2} 2_{02}-1_{11} 5 / 2-3 / 2^{3}$. The contributions by most of these species $\left(\mathrm{NH}, \mathrm{NH}_{2}, \mathrm{NH}_{3}\right.$, and $\mathrm{CH}$ ) are included in our model for $\mathrm{OH}^{+}$below (\$3.1.1).

\subsubsection{Main characteristics of the $\mathrm{OH}^{+}$lines}

The $\mathrm{OH}^{+}$spectra displayed in Fig. 1 show clear indications of extremely high-velocity outflowing gas, together with absorption near systemic velocities (as found for OH; GA14, GA17). In the excited lines observed with PACS, the clearest outflow signatures are seen in the relatively strong $2_{2}-1_{1}$ and $2_{3}-1_{2}$ lines, obtained with high signal-to-noise ratios (SNR; Fig. 1b). These lines have lower level energies of $E_{\text {lower }} \approx 50 \mathrm{~K}$ (see Fig. 1 in GA13 for energy level diagrams for the three O-bearing molecular ions). The relatively weak $\mathrm{OH}^{+} 2_{2}-1_{2}$ and $2_{1}-1_{0}$ lines at $\sim 148 \mu \mathrm{m}$ also show clear indication of blueshifted absorption (Fig. 1c). The blueshifted line wings of the uncontaminated $2_{2}-1_{1}$ $152.4 \mu \mathrm{m}$ and $2_{1}-1_{0} 148.7 \mu \mathrm{m}$ transitions, showing absorption out to $\approx-1000 \mathrm{~km} \mathrm{~s}^{-1}$ from the line center, closely match the line wings of the (also excited) OH84 and OH65 line profiles (Fig. 2). At around systemic velocities, the $\mathrm{OH}^{+}$ $2_{2}-1_{1}$ absorption also closely matches the $\mathrm{OH} 84$ line profile, including the peak absorption blueshift of $\sim-120 \mathrm{~km} \mathrm{~s}^{-1}$, while the optically thinner $\mathrm{OH}^{+} 2_{1}-1_{0}$ line better matches the $\mathrm{OH} 65$ profile. This strongly suggests that both species are formed in the same gas components. The $\mathrm{OH}^{+} 2_{3}-1_{2}$ line profile also shows blueshifted absorption reaching velocities of $-1200 \mathrm{~km} \mathrm{~s}^{-1}$, merging with the $2_{2}-1_{1}$ line (Fig. 1b).

The $3_{3}-2_{2}$ and $3_{4}-2_{3}$ fine-structure components (Fig. 1d), with $E_{\text {lower }} \approx 140 \mathrm{~K}$, also show indications of blue absorption, as the potential contamination by $\mathrm{NH}_{3}$ to the blueshifted wing of the $3_{3}-2_{2}$ line is expected to be weak (\$3). No clear blueshifted absorption in the $\mathrm{OH}^{+}$profiles is seen in the $4_{J}-3_{J^{\prime}}$ transitions (Fig. 1e, $E_{\text {lower }} \approx 280 \mathrm{~K}$ ).

The ground-state $\mathrm{OH}^{+} 1_{J}-0_{1}$ lines detected with SPIRE also show evidence for outflowing gas (Fig. 1h), with the emission above the continuum redshifted in the three lines relative to the expected line centers, and also with clear indications of blueshifted absorption. The latter is seen in the $1_{2}-0_{1}$ line profile although $\mathrm{NH}$ probably also contributes to the broad absorption feature $(\S 3)$, and in the $1_{1}-0_{1}$ line

\footnotetext{
${ }^{3}$ First detected in the ISM by van Dishoeck et al. (1993), $\mathrm{NH}_{2}$ has a spectrum similar to $\mathrm{H}_{2} \mathrm{O}^{+}$with the rotational levels of the asymmetric rotor split into 2 fine-structure levels due to the unpaired electronic spin of $1 / 2$.
}

profile in spite of the proximity of the CO 9-8 line. Some hints of absorption are also observed in the weakest transition, $1_{0}-0_{1}$.

As noted above, the strongest low-lying $2 J_{J}-1_{J^{\prime}}$ lines in Fig. 1b-c show the peak absorption blueshifted by $\sim 120$ $\mathrm{km} \mathrm{s}^{-1}$, while the higher-lying lines peak at central velocities. This behavior resembles that found in the $\mathrm{OH}$ lines of Mrk 231 (GA14), where the peak absorption of the OH84 transition is also blueshifted but the higher-lying lines (e.g., OH65 and OH71) peak closer to the systemic velocities (see also Fig. 2). In addition, no excited $\mathrm{OH}^{+}$lines show redshifted emission above the continuum. This behavior is also similar to that seen in the $\mathrm{OH}$ line profiles. Only the groundstate $\mathrm{OH} 119$ and $\mathrm{OH} 79$ doublet profiles show prominent redshifted emission (GA14, GA17), just as only the ground-state lines of $\mathrm{OH}^{+}$show redshifted emission features (Fig. 1h; van der Werf et al. 2010). Finally, comparison of the $\mathrm{OH}^{+} \mathrm{PACS}$ spectra in Mrk 231 and Arp 220 indicates that the absorption strength (relative to the continuum) at systemic velocities is similar in both sources (Fischer et al., in prep.), the important difference being the high-velocity blueshifted absorption in Mrk 231 that is absent in Arp 220.

Because of the non-Gaussian shapes of the $\mathrm{OH}^{+}$lines, we measured the equivalent widths and fluxes of the PACS lines in Table 2 by numerically integrating the line shapes over two velocity ranges, around systemic velocities and, when detected, in the blueshifted wings. Based on the change in the slope of the $\mathrm{OH}^{+}$PACS spectra (see e.g. Fig. 2), we adopted blueshifted and systemic velocity ranges of $[-1000,-300]$ and $[-300,200] \mathrm{km} \mathrm{s}^{-1}$, respectively; the latter upper limit is set to avoid strong contamination by neighboring lines on the red side. The $1 \sigma$ uncertainties of the equivalent widths were evaluated from the $\sigma_{\mathrm{rms}}^{\text {norm }}$ noise of the continuum-normalized PACS spectra as $\sigma_{\text {rms }}^{\text {norm }} \times \Delta V / n^{1 / 2}$, where $\Delta V$ and $n$ are the velocity coverage and number of channels over which $W_{\mathrm{eq}}$ is measured (González-Alfonso et al. 2015). Line detection is established at $\geq 3 \sigma$ level. The equivalent widths and fluxes of the $\mathrm{OH}^{+}$ground-state lines (Table 3) as measured with SPIRE are also integrated over two velocity ranges, corresponding to the emission and absorption features. We adopted a common velocity range $\left([-100,850] \mathrm{km} \mathrm{s}^{-1}\right)$ for the emission features, enabling comparison of the strengths of the $\mathrm{OH}^{+} 1_{J}-0_{1}$ emission lines. Uncertainties for the equivalent widths of the ground-state $\mathrm{OH}^{+}$lines are dominated by the remaining ripples of the apodized spectrum, rather than by random noise. A typical semi-cycle of the ripples has a width similar to that of the observed lines, so that we conservatively adopt the area of one semi-cycle (averaged over several cycles measured close to the line) as the $1 \sigma$ uncertainty of the equivalent width, and adopt a detection criterion of $\geq 2 \sigma$. 


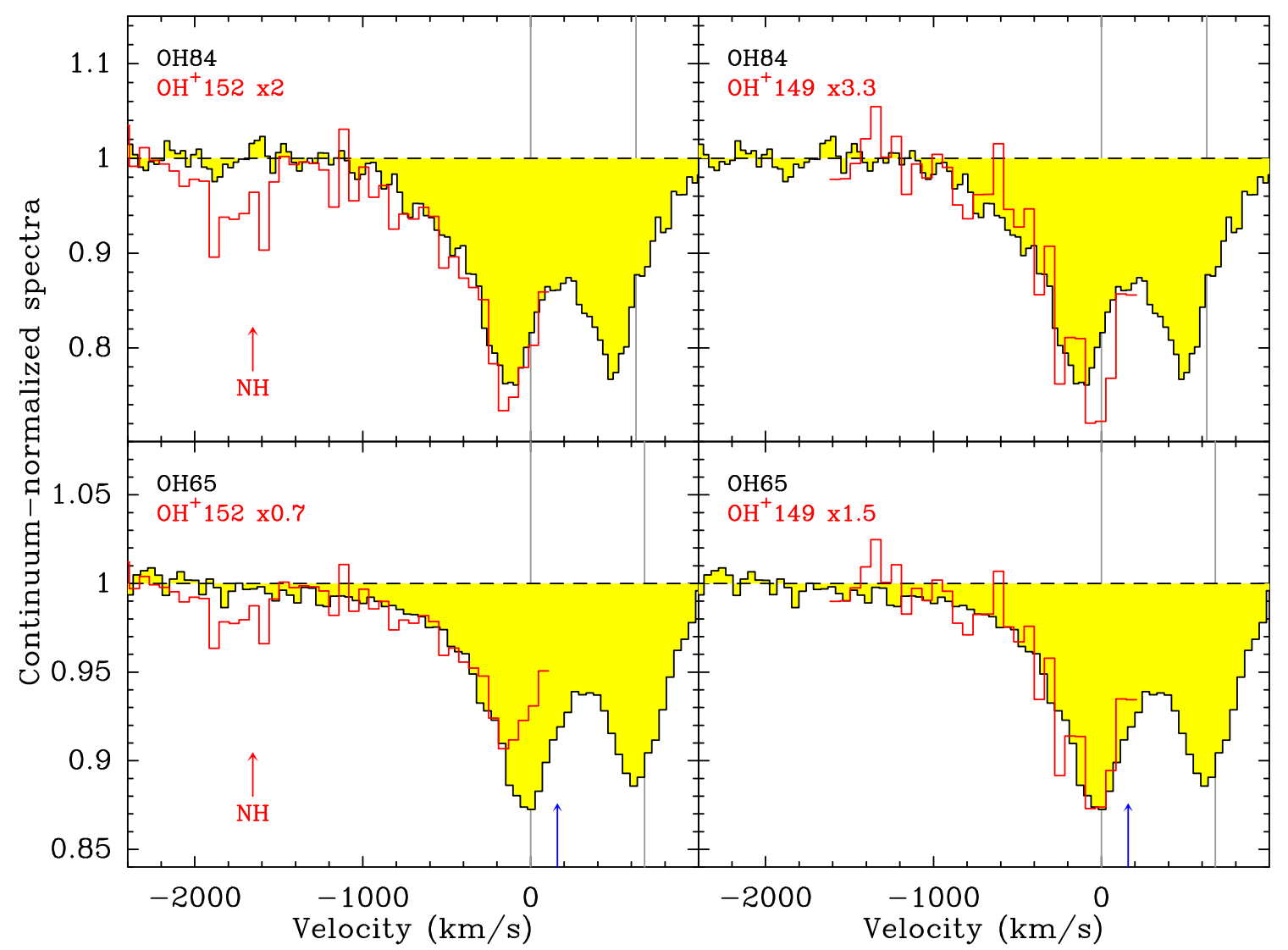

Figure 2. Comparison of the line shapes of $\mathrm{OH}^{+} 2_{2}-1_{1}$ at $152.4 \mu \mathrm{m}$ (left panels, in red) and $\mathrm{OH}^{+} 2_{1}-1_{0}$ at $148.7 \mu \mathrm{m}$ (right panels) with the excited $\mathrm{OH}$ at 84 and $65 \mu \mathrm{m}$ (yellow histograms). The two vertical gray lines in each panel indicate the positions of the doublet components of the OH84 and OH65 transitions, and the blue arrows in the lower panels indicate potential contamination to the OH65 spectrum by the very excited $\mathrm{H}_{2} \mathrm{O} 6_{25}-5_{14}$ line. The good match between the $\mathrm{OH}^{+} 152$ and $\mathrm{OH} 84$, and between the $\mathrm{OH}^{+} 149$ and $\mathrm{OH} 65$ lines, at both central and blueshifted velocities indicates that the lines of both species are generated in the same regions.

We caution that with the current spectral resolution of the apodized spectrum $\left(\sim 650 \mathrm{~km} \mathrm{~s}^{-1}\right)$ the $\mathrm{OH}^{+}$ground-state emission features will be contaminated by the absorption and vice versa, and that the ripples in the spectrum (Fig. 1h) make our flux measurements somewhat uncertain (Table 3). Nevertheless, optically thin emission would imply relative fluxes for the $1_{0}-0_{1}, 1_{1}-0_{1}$, and $1_{2}-0_{1}$ lines of $1: 3: 5$, which are the degeneracies of the upper levels ${ }^{4}$. The $\mathrm{OH}^{+} 1_{0}-0_{1}$ and $1_{1}-0_{1}$ emission features show, however, similar fluxes, which are a factor $\sim 1.4$ weaker than the $1_{2}-0_{1}$ emission (Table 3). This suggests both strong contamination of the

\footnotetext{
${ }^{4}$ This strictly applies to collisional excitation and, most probably, to the emission associated with formation pumping; if the $\mathrm{OH}^{+}$scatters continuum radiation, the contrast between the $1_{0}-0_{1}$ component and the other two would be even higher due to the decreasing continuum strength with increasing wavelength.
}

$1_{0}-0_{1}$ line by $\mathrm{NH}_{2}{ }^{5}$, and significant optical depth effects in the $1_{J}-0_{1}$ lines.

\subsection{The $\mathrm{H}_{2} \mathrm{O}^{+}$spectra}

In GA13, the strongest, lowest-lying, and uncontaminated lines of $\mathrm{H}_{2} \mathrm{O}^{+}$in the far-IR were identified and analyzed in Arp 220 and NGC 4418. We show in Fig. 3a-f ${ }^{6}$ the PACS spectra of Mrk 231 around the same lines (the spectrum in panel a is contaminated by $\mathrm{NH}_{2}$ ). Evidence for absorption in Mrk 231 is found in three $\mathrm{H}_{2} \mathrm{O}^{+}$transitions: the $22_{21}-1_{10} 5 / 2-3 / 2$ para- $\mathrm{H}_{2} \mathrm{O}^{+}$line (Fig. $3 \mathrm{~b}$ ), and the $3_{13}-$ $2_{02} 5 / 2-3 / 2$ and $7 / 2-5 / 2$ ortho lines (panel e). There may also be some hints of absorption in the $3_{22}-2_{11} 5 / 2-3 / 2$ line

\footnotetext{
5 The emission feature at $907.4 \mathrm{GHz}$ in the $\mathrm{OH}^{+} 1_{0}-0_{1}$ spectrum of Arp 220 shown by Rangwala et al. (2011) is most probably dominated by $\mathrm{NH}_{2} 2_{02}-1_{11} 5 / 2-3 / 2$, as the $3 / 2-1 / 2$ component at $902.2 \mathrm{GHz}$ is clearly seen in their spectrum.

${ }^{6}$ In Mrk 231, the ground-state para- $\mathrm{H}_{2} \mathrm{O}^{+} 2_{12}-1_{01}$ lines at rest wavelengths of 182.9 and $184.3 \mu \mathrm{m}$ were not covered by our full PACS scan of Mrk 231 as they lie in the $\gtrsim 190 \mu \mathrm{m}$ leakage region.
} 
(panel c), but the 7/2-5/2 fine-structure component, which is expected to be stronger, is not detected (panel d).

The detected $\mathrm{H}_{2} \mathrm{O}^{+}$lines peak close to systemic velocities with no evidence for blueshifted absorption wings. In comparison with Arp 220, the $22_{21}-1_{10} 5 / 2-3 / 2$ and $33_{13}-$ $2_{02} 5 / 2-3 / 2$ lines have similar absorption strengths (relative to the continuum) in both sources, but the $3_{13}-2_{02} 7 / 2-5 / 2$ line is stronger in Arp 220.

The SPIRE spectrum shows strong emission in the groundstate $\mathrm{H}_{2} \mathrm{O}^{+} 1_{11}-0_{00} 1 / 2-1 / 2$ line (Fig. $3 \mathrm{~g}$ ), which is surprising given the weakness of the absorption lines seen with PACS. While the emission feature has a peak strength similar to that of the $\mathrm{OH}^{+} 1_{1}-0_{1}$ line (Fig. 1h), it shows no measurable absorption at blueshifted velocities - in contrast with the $\mathrm{OH}^{+}$ground-state lines even though the continuum is stronger at the wavelength of the $\mathrm{H}_{2} \mathrm{O}^{+}$line. On the other hand, the $\mathrm{H}_{2} \mathrm{O}^{+} 1_{11}-0_{00} 1 / 2-1 / 2$ spectral feature shows a prominent redshifted shoulder, which is attributable to the $\mathrm{H}_{2}^{18} \mathrm{O} 3_{21}-3_{12}$ line. This identification relies on the facts that the corresponding $3_{21}-3_{12}$ line of the main isotopologue $\mathrm{H}_{2}^{16} \mathrm{O}$ is strong in Mrk 231 (GonzálezAlfonso et al. 2010), and that ${ }^{18} \mathrm{O}$ is extremely abundant in this source as inferred from PACS observations and modeling of $\mathrm{OH}$ and ${ }^{18} \mathrm{OH}$ (Fischer et al. 2010, GA14). On the other hand, the feature identified with $\mathrm{H}_{2} \mathrm{O}^{+}$cannot be a blueshifted feature of the $\mathrm{H}_{2}^{18} \mathrm{O}$ line, because the corresponding $\mathrm{H}_{2}^{16} \mathrm{O}$ line shows no blueshifted shoulder. Unfortunately, the $\mathrm{H}_{2} \mathrm{O}^{+} 1_{11}-0_{00} 3 / 2-1 / 2$ fine-structure component (Fig. $3 \mathrm{~g}$ ) is blended with the ground-state $1_{11}-0_{00}$ line of $\mathrm{H}_{2}^{16} \mathrm{O}$, and cannot confirm the interpretation of the $1 / 2-1 / 2$ line. Our searches of the CDMS and JPL catalogs, however, do not reveal plausible alternatives to the quoted $\mathrm{H}_{2} \mathrm{O}^{+}$and $\mathrm{H}_{2}^{18} \mathrm{O}$ lines to explain the spectral emission at 263-264 $\mu \mathrm{m}$.

Tables 2 and 3 list the equivalent widths and fluxes of the $\mathrm{H}_{2} \mathrm{O}^{+}$PACS and SPIRE lines, respectively. Uncertainties were calculated in the same way as for the $\mathrm{OH}^{+}$lines (\$2.1.2). The flux of the ground-state $1_{11}-0_{00} 1 / 2-1 / 2$ line was integrated up to $400 \mathrm{~km} \mathrm{~s}^{-1}$ to avoid strong contamination by the blended $\mathrm{H}_{2}^{18} \mathrm{O}$ line.

\subsection{The $\mathrm{H}_{3} \mathrm{O}^{+}$spectra}

There is very little evidence for $\mathrm{H}_{3} \mathrm{O}^{+}$in the PACS and SPIRE spectra of Mrk 231. A search for the transitions that were detected in Arp 220 and/or NGC 4418 (GA13) resulted mostly in negative results. The lower signal-to-noise ratio in some ranges of the Mrk 231 spectrum in comparison with that of Arp 220, cannot on its own account for this difference. In contrast to Arp 220, no pure-inversion metastable lines are detected in Mrk 231. We show in Fig. 4 the PACS spectrum of Mrk 231 around the $\mathrm{H}_{3} \mathrm{O}^{+}$lines that are expected to be strongest as seen in the PACS spectrum of Arp 220. The only transition that shows some hints of absorption is the ortho-
$\mathrm{H}_{3} \mathrm{O}^{+} 4_{3}^{-}-3_{3}^{+}$. We use these spectra in $\S 3$ to establish an upper limit for the $\mathrm{OH}^{+} / \mathrm{H}_{3} \mathrm{O}^{+}$ratio. The ortho- $\mathrm{H}_{3} \mathrm{O}^{+}$groundstate $0_{0}^{-}-1_{0}^{+}$line at $304.45 \mu \mathrm{m}$ is also absent in Mrk 231 (not shown). Upper limits for the equivalent widths and fluxes of the $\mathrm{H}_{3} \mathrm{O}^{+}$PACS lines ( $2 \sigma$, calculated in the same way as for the $\mathrm{OH}^{+}$lines) are listed in Table 2.

\section{ANALYSIS}

\subsection{Radiative transfer models}

GA17 modeled the $\mathrm{OH}$ line profiles in Mrk 231 with three components, (i.e., a simpler scheme than the four-component model of GA14). The "CORE" component, which hereafter will be called the quiescent component (QC), physically represents the $\sim 100 \mathrm{pc}$-scale torus around the AGN detected in $\mathrm{OH}$ at centimeter wavelengths (Klöckner et al. 2003), and is intended to account for the line absorption in the nuclear region of the galaxy close to systemic velocities. The line wings and $\mathrm{P}-\mathrm{Cygni}$ profiles observed in $\mathrm{OH}$ require two additional "ENVELOPE" components to reproduce spectral features arising in the outflowing molecular gas. One of the outflowing components is compact, as it accounts for the absorption in the excited OH84 and OH65 doublets. It has maximum velocities of $\gtrsim 1000 \mathrm{~km} \mathrm{~s}^{-1}$, and will be referred to as the high-velocity component (HVC). The other outflow component has lower maximum velocities and is primarily detected in the ground-state OH119 and OH79 doublets; it will be referred to as the low-excitation component (LEC). In order to account for most of the absorption and emission in $\mathrm{OH} 119$ and OH79, the LEC must be more extended than the QC and HVC components, but it contributes little to the excited OH84 and OH65 doublets. The spectra of the Obearing molecular ions reported in the present work are modeled and interpreted in terms of this physically and spectrally motivated 3-component model. By using the same physical parameters for these components as in GA17, our only free parameters here are the abundances of $\mathrm{OH}^{+}, \mathrm{H}_{2} \mathrm{O}^{+}$, and $\mathrm{H}_{3} \mathrm{O}^{+}$ relative to $\mathrm{H}$ nuclei (regardless of whether the hydrogen is in atomic or molecular form), i.e., $X\left(\mathrm{OH}^{+}\right), X\left(\mathrm{H}_{2} \mathrm{O}^{+}\right)$, and $X\left(\mathrm{H}_{3} \mathrm{O}^{+}\right)$in each component (QC, HVC, and LEC). Because the physical model (continuum optical depths, dust temperatures, sizes, densities, linewidths, velocity gradients, molecular distribution) takes exactly the same values for all modeled species, the abundance ratios are less dependent on intrinsic model uncertainties.

The molecules and dust are mixed in the CORE components, and hence the absolute abundances for the QC are inferred from the molecular column density and the continuum optical depth at $100 \mu \mathrm{m}, \tau_{100}$, by assuming a standard gasto-dust ratio of 100 by mass (see also González-Alfonso et al. 2012; Falstad et al. 2015; Stone et al. 2017). For the outflowing components, however, the optical depth of the mixed dust is not well constrained (the absorption is primarily pro- 

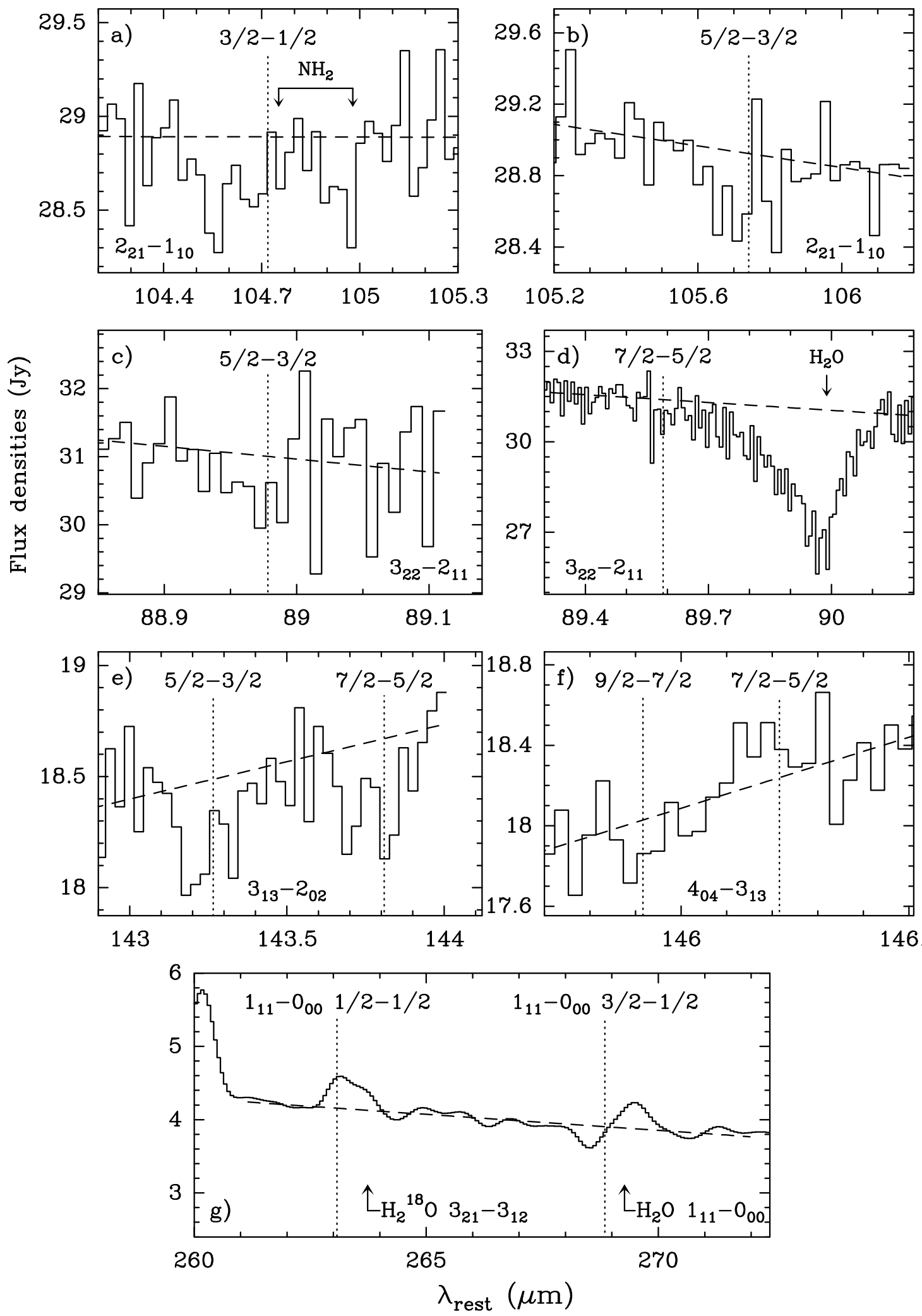

Figure 3. The observed spectra around the uncontaminated and presumably strongest (as seen in Arp 220, GA13) $\mathrm{H}_{2} \mathrm{O}^{+}$lines in $\mathrm{Mrk}^{231}$, with the adopted baselines (dashed lines). The rotational transitions are indicated at the bottom of each panel, and the fine-structure components are indicated above the spectra. The rest wavelengths are calculated with respect to the systemic redshift of $z=0.04218$. The absorption shown in panel a is probably contaminated by $\mathrm{NH}_{2} 3_{22}-2_{11} 5 / 2-3 / 2$ and $7 / 2-5 / 2$. The ground-state $\mathrm{H}_{2} \mathrm{O}^{+} 1_{11}-0_{00} 1 / 2-1 / 2$ line in panel g has a redshifted shoulder attributable to $\mathrm{H}_{2}^{18} \mathrm{O}$, and the $3 / 2-1 / 2$ fine-structure component is strongly blended with the para- $\mathrm{H}_{2} \mathrm{O} 1_{11}-0_{00}$ ground-state line. 


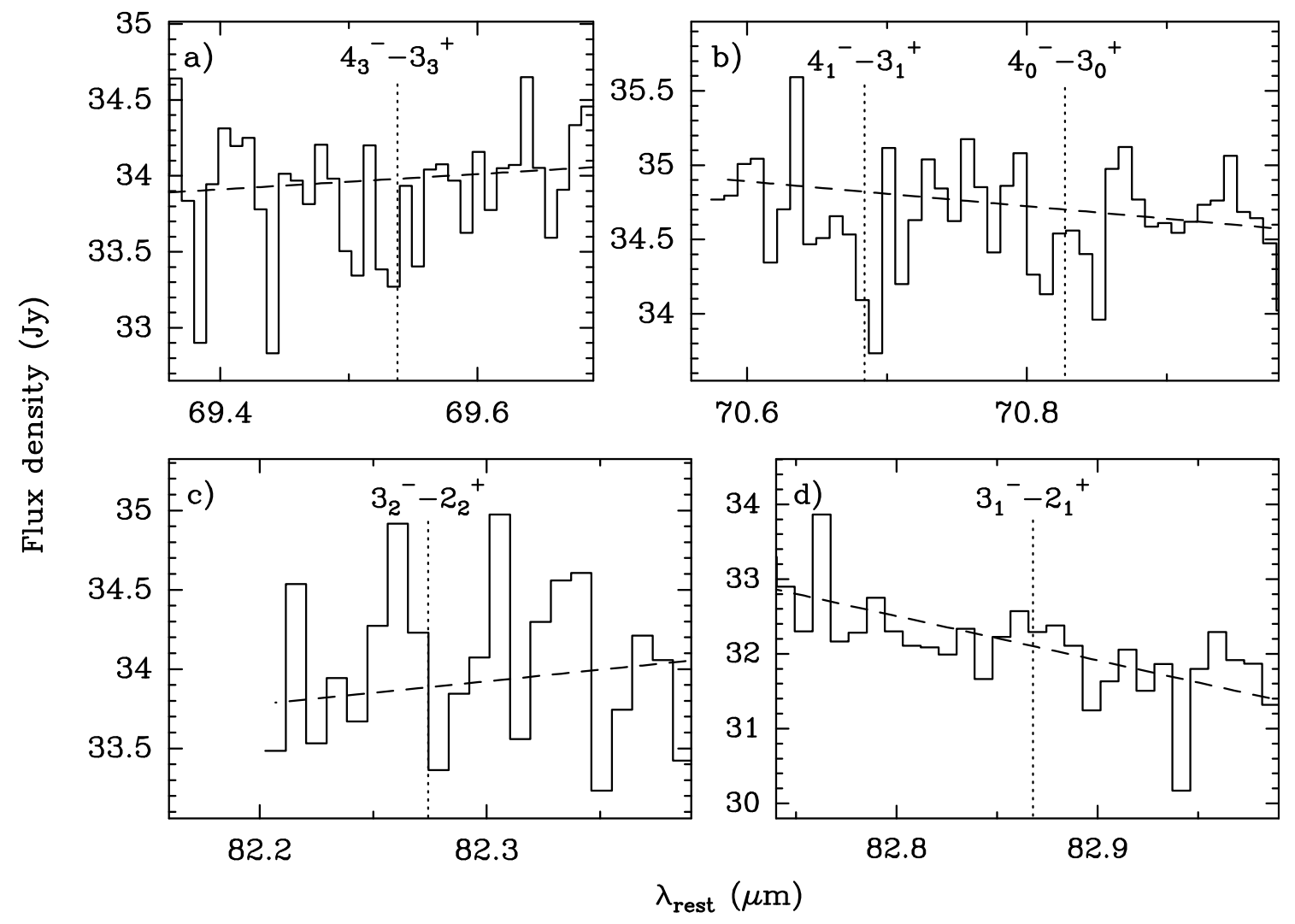

Figure 4. The observed spectra around the uncontaminated and presumably strongest (as seen in NGC 4418 and $\mathrm{Arp} 220$, GA13) $\mathrm{H}_{3} \mathrm{O}^{+}$lines in Mrk 231, with the adopted baselines (dashed lines). The rest wavelengths are calculated with respect to the systemic redshift of $z=0.04218$. There are only some hints of absorption in the lines shown in panels a and $b$, and no hints of absorption in the pure-inversion metastable lines (not shown). Likewise, the ground-state ortho line $0_{0}-1_{0}$ line at $304.45 \mu \mathrm{m}$ is not detected (not shown). 

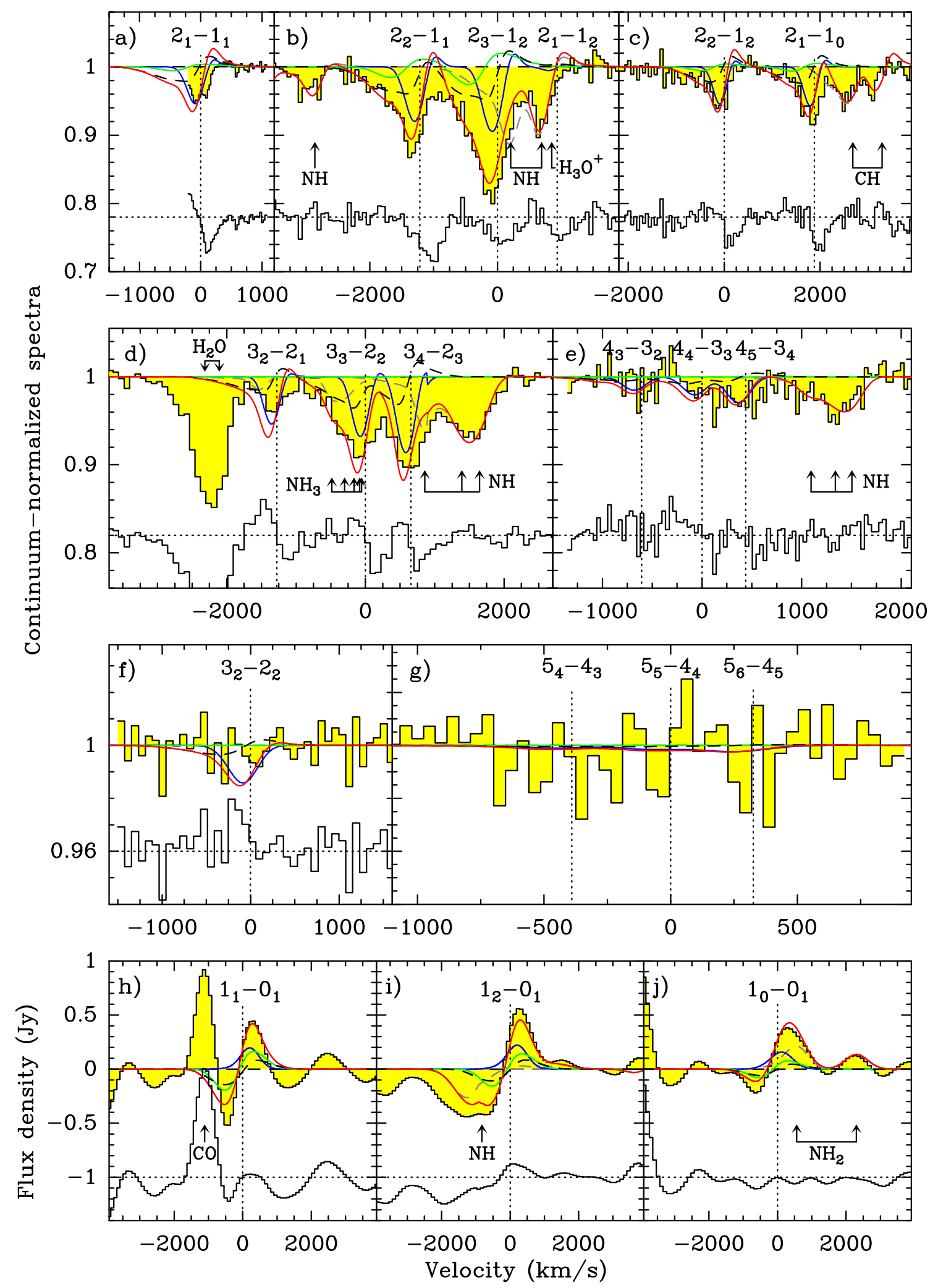

Figure 5. Model fit for the $\mathrm{OH}^{+}$lines in Mrk 231. The blue, dashed-black, and green curves show the contributions by the quiescent component (QC), the high-excitation outflow component (HVC), and the low-excitation component (LEC), respectively. The model includes the estimated contributions by $\mathrm{NH}$ (panels b, d, e, i), $\mathrm{NH}_{2}$ (panel j), $\mathrm{NH}_{3}$ (panel d), and $\mathrm{CH}$ (panel c), shown as gray-dashed curves. The red curve indicates the summed profiles from all three components, and the black histograms at the bottom of each panel (except $\mathrm{g}$ ) show the residuals (data-model) vertically shifted by the value indicated with the horizontal-dotted lines. The physical parameters used for the QC and HVC models are the same as in the model for $\mathrm{OH}$ in GA17. In panel a, the $\mathrm{OH}^{+} 2_{1}-1_{1}$ line is truncated at blueshifted velocities due to the proximity of the [C II] $158 \mu \mathrm{m}$ line. 


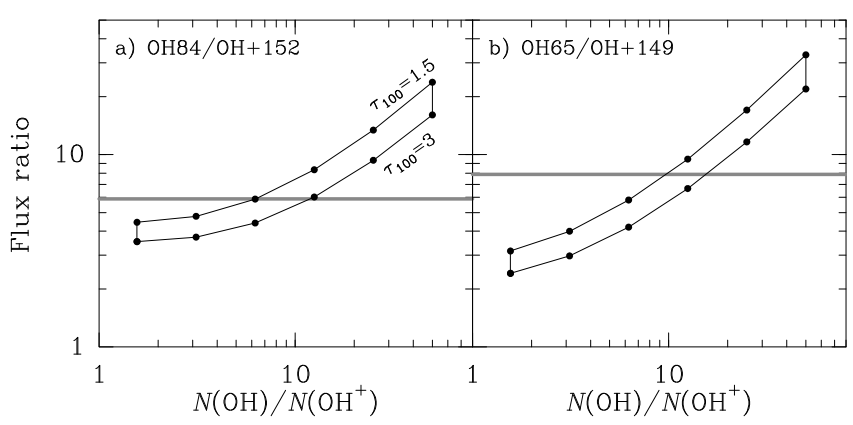

Figure 6. a) The $\mathrm{OH} 84 / \mathrm{OH}+152$ and b) the $\mathrm{OH} 65 / \mathrm{OH}+149$ line flux ratios (both in $\mathrm{erg} \mathrm{s}^{-1} \mathrm{~cm}^{-2}$ ), integrated between -1000 and $-300 \mathrm{~km} \mathrm{~s}^{-1}$, as a function of the $\mathrm{OH} / \mathrm{OH}^{+}$column density ratio. Model results for the high-velocity component (HVC) are shown for optical depths of the continuum source at $100 \mu \mathrm{m}$ of $\tau_{100}=1.5$ and 3 . The horizontal lines show the values measured in Mrk 231, indicating $N(\mathrm{OH}) / N\left(\mathrm{OH}^{+}\right) \approx 10$ in the HVC. Therefore, $X\left(\mathrm{OH}^{+}\right)>10^{-7}$ for $X(\mathrm{OH})>10^{-6}$ in the excited, outflowing component.

duced by the continuum source behind the approaching gas), and an abundance $X(\mathrm{OH})=2.5 \times 10^{-6}$ was adopted in GA14 and GA17.

For a given species, the column densities associated with the QC, HVC, and LEC components are derived from a combination of kinematic and excitation characteristics. The spectral features (in absorption or emission) detected at central velocities are associated with the $\mathrm{QC}$. The line wings associated with excited lines are associated with the HVC, with specific predictions for the absorption and emission in the ground-state lines. Therefore, the line wings associated with the ground-state lines, which similarly to $\mathrm{OH}$ arise mainly in a more extended and less excited component, are reproduced once the LEC is included in the full model.

\subsubsection{Models for $\mathrm{OH}^{+}$}

Figure 5a-g presents our best model fit to the $\mathrm{OH}^{+}$line profiles observed by PACS and Table 4 lists the inferred $\mathrm{OH}^{+}$ column densities and abundances. Besides the pumping generated by the strong far-IR radiation field, we also include collisional excitation with $\mathrm{H}$ atoms (Lique et al. 2016) and with electrons (van der Tak et al. 2013) assuming a fractional abundance $X\left(\mathrm{e}^{-}\right)=10^{-3}$ (see §3.2.1); collisional excitation with $\mathrm{H}$ is found to dominate. In our model fitting, we also include predictions for $\mathrm{NH}, \mathrm{NH}_{2}, \mathrm{NH}_{3}$, and $\mathrm{CH}$. We find similar $\mathrm{NH}$ and $\mathrm{OH}^{+}$column densities for the $\mathrm{QC}$, and we adopt the same column for $\mathrm{NH}$ as for $\mathrm{OH}^{+}$in the outflowing components as well. Our model for $\mathrm{NH}_{2}$ and $\mathrm{NH}_{3}$ only includes the prediction for the $\mathrm{QC}$, based on the fits to other unblended $\mathrm{NH}_{2}$ and $\mathrm{NH}_{3}$ lines detected across the PACS spectrum (Fischer et al. in prep).

The PACS data mainly constrain the column densities of the QC and $\mathrm{HVC}$ components, where $\mathrm{OH}^{+}$is excited by the far-IR field. Model results for the HVC are shown in Fig. 6, which compares the predicted $\mathrm{OH} 84 / \mathrm{OH}^{+} 152$ and
$\mathrm{OH} 65 / \mathrm{OH}^{+} 149$ flux ratios in the blueshifted wings (the line observations that are compared in Fig. 2) with the measured values, indicating $\mathrm{OH} / \mathrm{OH}^{+}$column density ratios of $\sim 10$ in the excited outflow component. For the $\mathrm{QC}$, the $\mathrm{OH} / \mathrm{OH}^{+}$ ratio is $\sim 20$ (Table 4 ). These column density ratios indicate very high $\mathrm{OH}^{+}$abundances, $\sim 10^{-7}$ in the $\mathrm{QC}$ and even higher in the HVC for $X(\mathrm{OH})>10^{-6}$ (Stone et al. 2017).

Our model for the QC, which includes an expansion velocity of $100 \mathrm{~km} \mathrm{~s}^{-1}$ (GA17), reproduces the blueshift observed in the $\mathrm{OH}^{+} 2{ }_{J}-1_{J^{\prime}}$ lines but also predicts a blueshift in higher-lying lines that is not observed. The quality of the fit is nevertheless reasonable for most lines at central and blueshifted velocities, though our spherically symmetric models predict significant redshifted reemission above the continuum in some $2_{J}-1_{J^{\prime}}$, which is not detected (see the residuals in Fig. 5). In addition, an observed "bridge" of absorption joins the $2_{3}-1_{2}$ and the $2_{2}-1_{1}$ lines (panel $b$ ), and the $3_{3}-2_{2}$ and the $3_{2}-2_{1}$ lines (panel d) as well, that cannot be reproduced. These deviations of the spectral fits from the data may indicate a departure from spherical symmetry, with the outflow mainly focussed along the line of sight (GA14), or a redshifted absorption component that could represent low-velocity inflowing gas.

Figs. $5 \mathrm{~h}-\mathrm{j}$ compare the profiles of the ground-state $\mathrm{OH}^{+}$ lines observed with SPIRE with the predictions of our composite model. We have not attempted to correct the spectrum for the residual ripples in the FTS apodized spectrum, and thus our results are only approximate. Two main conclusions can nevertheless be drawn from this comparison. First, the HVC and the QC together cannot account for either the absorption or the emission features observed in the $\mathrm{OH}^{+} 1_{J}-0_{1} \mathrm{P}$-Cygni profiles, so that we require the inclusion in the model of the LEC with a high $\mathrm{OH}^{+}$column of $\sim 10^{16}$ $\mathrm{cm}^{-2}$. This is a factor of only $\sim 5$ lower than the column derived for $\mathrm{OH}$ in the same LEC, and thus a very high $\mathrm{OH}^{+}$ abundance $\left(>2 \times 10^{-7}\right)$ is favored. Second, the observed $\mathrm{OH}^{+}$redshifted emission features, including in particular that of the uncontaminated $1_{2}-0_{1}$ component (Fig. 5i), require high volume densities (i.e. collisional excitation) at least for the QC. Otherwise, the model for the QC would predict the $\mathrm{OH}^{+}$ground-state lines in absorption close to systemic velocities, which is not observed, and the emission features would be underpredicted. In the model shown in Fig. 5, the density decreases from $\sim 10^{6} \mathrm{~cm}^{-3}$ in the innermost regions of the QC to $\sim 1.5 \times 10^{5} \mathrm{~cm}^{-3}$ in the external parts.

Even with high $\mathrm{OH}^{+}$column densities in both outflowing components, Figs. $5 \mathrm{~h}-\mathrm{j}$ show that the absorption features of the ground-state $\mathrm{OH}^{+}$lines, and in particular the $1_{0}-0_{1}$ one, are underestimated by the model. The three lines are optically thick in the three model components, so that an increase in column density would hardly improve the fit. The most probable reason for this discrepancy is the model un- 


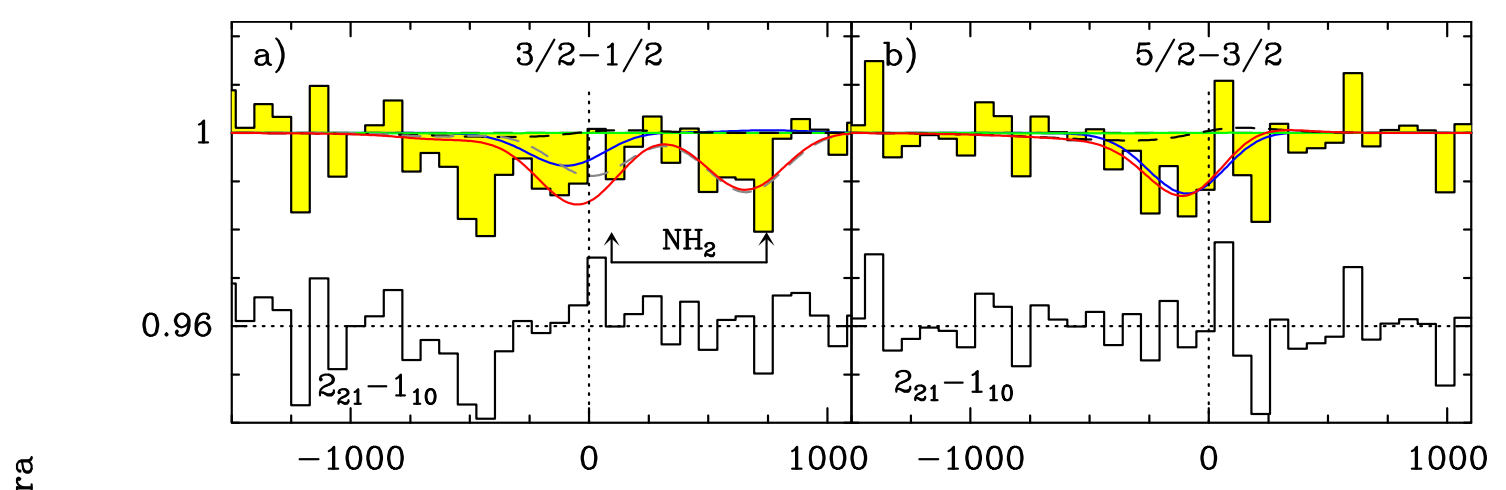

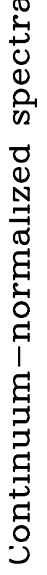
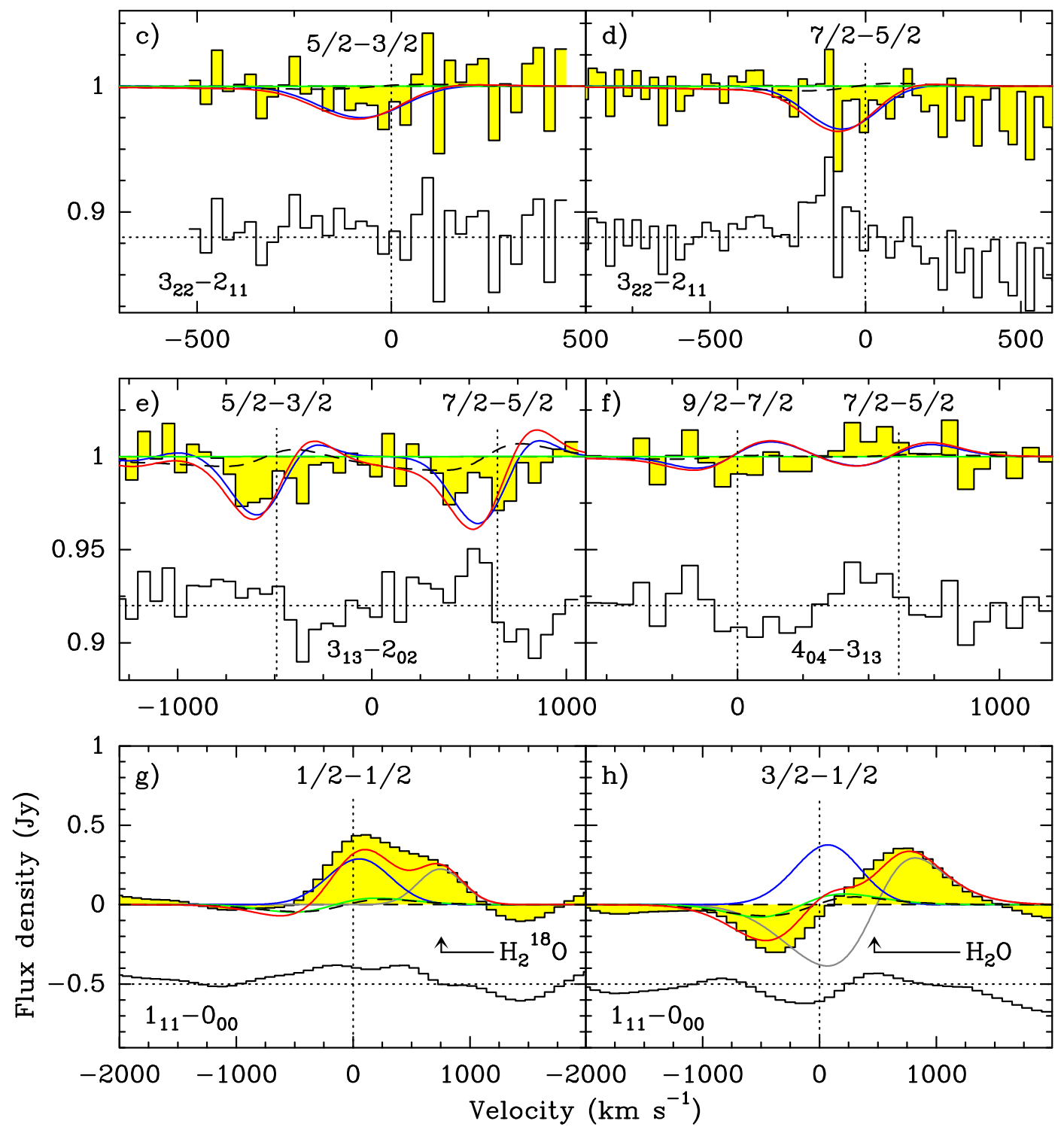

Figure 7. A model for the $\mathrm{H}_{2} \mathrm{O}^{+}$lines in Mrk 231, which establishes a lower limit for the $\mathrm{OH}^{+} / \mathrm{H}_{2} \mathrm{O}^{+}$abundance ratio in both the QC (blue lines) and the HVC (dashed-black lines). The LEC is also included in green, but has a negligible effect on the PACS lines. The red curve indicates the summed profiles from all three components, and the black histograms at the bottom of each panel show the residuals (data-model) vertically shifted by the value indicated with the horizontal-dotted lines. We obtain $\mathrm{OH}^{+} / \mathrm{H}_{2} \mathrm{O}^{+} \gtrsim 4$ for the QC and $\mathrm{OH}^{+} / \mathrm{H}_{2} \mathrm{O}^{+} \gtrsim 10$ for the $\mathrm{HVC}(\mathrm{mostly}$ based on the detected lines in panel e). The physical parameters used for the QC and HVC models are the same as in the model for OH in GA17. Panel a includes a model for $\mathrm{NH}_{2}$ (the same as used in Fig. 5j), panel $\mathrm{g}$ includes $\mathrm{H}_{2}^{18} \mathrm{O}$, and panel $\mathrm{h}$ includes a model for $\mathrm{H}_{2} \mathrm{O}$ in the outflow (in gray). 


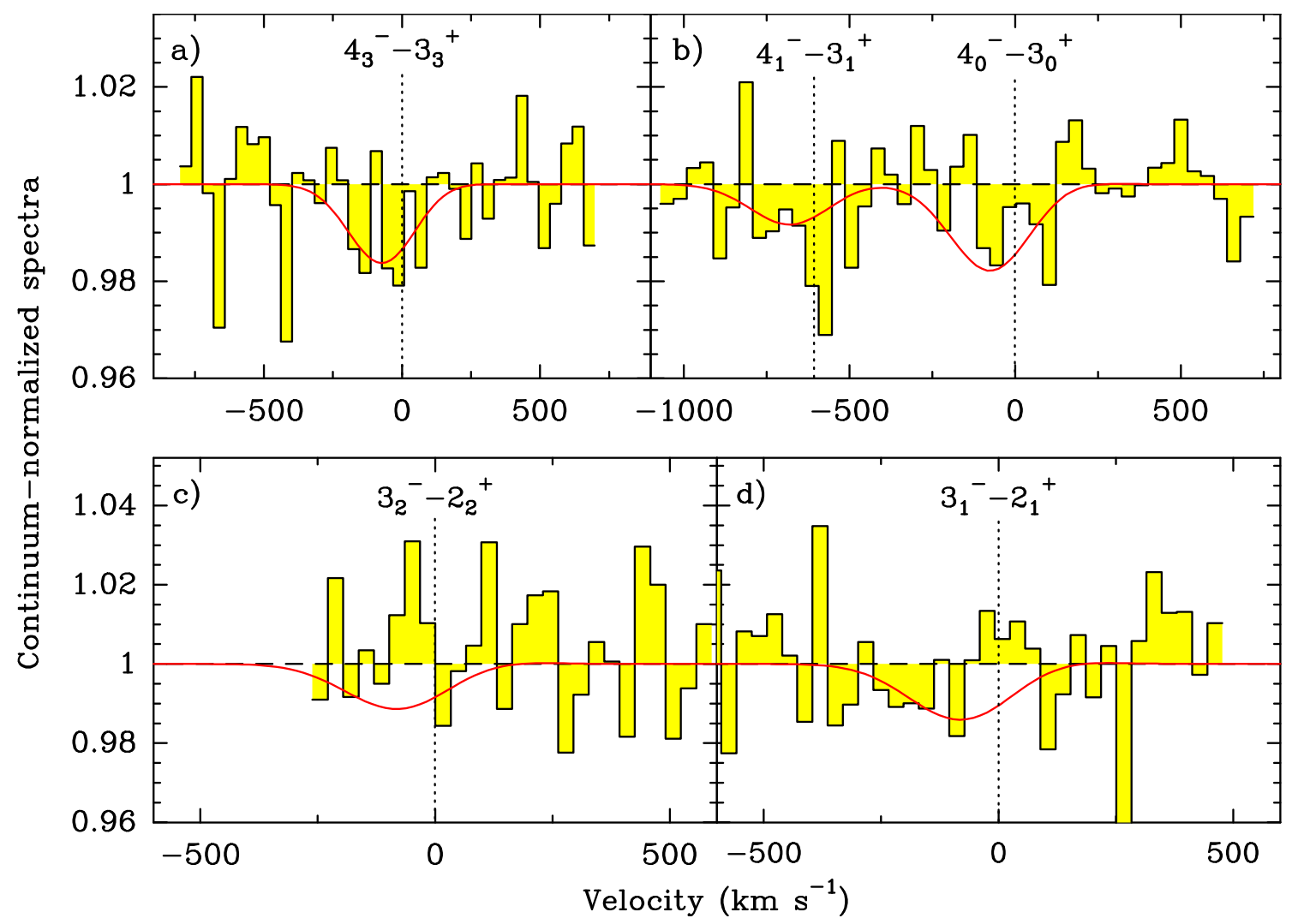

Figure 8. A model for the $\mathrm{H}_{3} \mathrm{O}^{+}$lines in Mrk 231, which establishes a lower limit for the $\mathrm{OH}^{+} / \mathrm{H}_{3} \mathrm{O}^{+}$abundance ratio in the QC (no significant limits are obtained for the outflowing components). We obtain $\mathrm{OH}^{+} / \mathrm{H}_{3} \mathrm{O}^{+} \gtrsim 4$ for the QC. The physical parameters used for the QC model are the same as in the model for $\mathrm{OH}$ in GA17. 
derestimation of the submillimeter continuum by a factor of $\approx 2$ at $300 \mu \mathrm{m}$; a moderate increase in $T_{\text {dust }}$ or in the continuum optical depth at submillimeter wavelengths would produce deeper absorption in the $1_{J}-0_{1}$ lines. On the other hand, we also note that the high densities inferred from the ground-state emission features, which are volume tracers as they probe deep into the buried nuclear regions, do not necessarily apply to the gas responsible for the absorption features in excited lines, which are surface tracers; absorption and emission features are indeed produced in different regions of the QC (see discussion in $\$ 3.2 .1$ ).

\subsubsection{Models for $\mathrm{H}_{2} \mathrm{O}^{+}$}

As noted in $\S 2.2$, the $\mathrm{H}_{2} \mathrm{O}^{+}$lines in Mrk 231 are weak and show absorption only at systemic velocities, with no evidence for blueshifted wings. Figures 7a-f show the predicted $\mathrm{H}_{2} \mathrm{O}^{+}$ (and $\mathrm{NH}_{2}$ in panel a) PACS lines of a model with $\mathrm{H}_{2} \mathrm{O}^{+}$column densities of $8.4 \times 10^{16} \mathrm{~cm}^{-2}$ and $3 \times 10^{15} \mathrm{~cm}^{-2}$ in the QC and the $\mathrm{HVC}$, respectively. These $\mathrm{H}_{2} \mathrm{O}^{+}$columns are considered upper limits: while the model for the QC approximately accounts for the $2_{21}-1_{10} 5 / 2-3 / 2$ and $322-2115 / 2-3 / 2$ lines (Fig. 7b,c), it overpredicts the detected $\mathrm{H}_{2} \mathrm{O}^{+} 3_{13}-2_{02}$ $7 / 2-5 / 2$ and 5/2-3/2 lines (Fig. 7e) and the undetected $3_{22}-2_{11} 7 / 2-5 / 2$ line (Fig. 7d). Therefore, a $\mathrm{H}_{2} \mathrm{O}^{+}$column of $(4.2-8.4) \times 10^{16} \mathrm{~cm}^{-2}$ is favored for the QC, and $N\left(\mathrm{H}_{2} \mathrm{O}^{+}\right) \lesssim 3 \times 10^{15} \mathrm{~cm}^{-2}$ is obtained for the HVC (Table 4).

With these moderate columns, we emphasize the surprisingly strong emission feature associated with the groundstate $\mathrm{H}_{2} \mathrm{O}^{+} 1_{11}-0_{00} 1 / 2-1 / 2$ line in the SPIRE spectrum. While the lack of apparent blueshifted absorption in this line in consistent with the low $\mathrm{H}_{2} \mathrm{O}^{+}$column density found for the HVC from the PACS lines, the emission feature would probably require either very high volume densities in the region of the QC where the line is generated, or formation pumping.

Unfortunately, no collisional rates between $\mathrm{H}_{2} \mathrm{O}^{+}$and $\mathrm{H}$ atoms are available, so we have attempted the following rough approach in order to check the consistency of the $\mathrm{H}_{2} \mathrm{O}^{+}$ identification and to establish a reference density based on an (uncertain) assumption on the collisional excitation of $\mathrm{H}_{2} \mathrm{O}^{+}$. The $\mathrm{OH}^{+}-\mathrm{H}$ de-excitation collisional rates reported by Lique et al. (2016) between the excited $\mathrm{OH}^{+}$levels and the ground-state $0_{1}$ state show little dependence on gas temperature and follow the approximate relationship $C_{u \rightarrow 0_{1}} / g_{0_{1}}=$ $8.5 \times 10^{-10} \times \Delta E^{-0.6} \mathrm{~cm}^{3} \mathrm{~s}^{-1}$, where $g_{0_{1}}=3$ is the degeneracy of the $0_{1}$ level and $\Delta E$ is the energy of the upper level. This expression holds up to $\Delta E>1000 \mathrm{~K}$, i.e. up to the highest energy levels considered by the authors, $N=7$. We have simply assumed that the above expression, with a (roundedoff) multiplicative constant of $10^{-9}$ (rather than $8.5 \times 10^{-10}$ ) to include collisions with electrons, yields characteristic values for the de-excitation rates between the O-bearing molecular ions in collisions with $\mathrm{H}$ atoms, and applied it to the de-excitation rates of $\mathrm{H}_{2} \mathrm{O}^{+}$transitions to the ortho $\left(0_{00}\right)$ and para $\left(1_{01} 3 / 2\right.$ and $\left.1 / 2\right)$ ground levels. The excitation rates are then calculated through detailed balance with an adopted gas temperature of $500 \mathrm{~K}$. For reference, the resulting collisional excitation rate from the $0_{00}$ level to the $1_{11} 1 / 2$ level is $\approx 8 \times 10^{-11} \mathrm{~cm}^{3} \mathrm{~s}^{-1}$. The collisional rates between excited levels are neglected.

Our overall model for $\mathrm{H}_{2} \mathrm{O}^{+}$also includes in Fig. $7 \mathrm{~g}$-h calculations for $\mathrm{H}_{2} \mathrm{O}$ and $\mathrm{H}_{2}^{18} \mathrm{O}$, the former based on the model reported in González-Alfonso et al. (2010) by also including an outflowing component; results for these species will be discussed in a future work. The main points relevant for the present study are $(i)$ to approximately reproduce the strength of the $\mathrm{H}_{2} \mathrm{O}^{+} 1_{11}-0_{00} 1 / 2-1 / 2$ line with our adopted collisional rates, a density of several $\times 10^{6} \mathrm{~cm}^{-3}$ in the QC is required, i.e. one order of magnitude higher than that inferred from the $\mathrm{OH}^{+}$ground-state lines; (ii) the model is roughly consistent with the lack of an emission feature in the $\mathrm{H}_{2} \mathrm{O}^{+} 1_{11}-0_{00} 3 / 2-1 / 2$ line (panel $\mathrm{h}$ ), due to cancellation by strong $\mathrm{H}_{2} \mathrm{O} 1_{11}-0_{00}$ absorption in the outflow; (iii) some contribution to the absorption wing observed in panel $\mathrm{h}$ ) can be attributed to $\mathrm{H}_{2} \mathrm{O}^{+}$, with the moderate column densities listed in Table 4.

We remark that the density inferred for the QC from the ground-state $\mathrm{H}_{2} \mathrm{O}^{+}$lines, several $\times 10^{6} \mathrm{~cm}^{-3}$, should only be taken as a qualitative confirmation of the results obtained for $\mathrm{OH}^{+}$, but the absorption lines seen by PACS and the emission lines seen by SPIRE do not sample the same regions (\$3.2.1). Our subsequent analysis (\$3.2) is based on the column densities inferred from the absorption lines, where the densities may be significantly lower.

\subsubsection{Models for $\mathrm{H}_{3} \mathrm{O}^{+}$}

As seen in $\$ 2.3$, little evidence is found in the PACS spectrum of Mrk 231 for $\mathrm{H}_{3} \mathrm{O}^{+}$, and an upper limit for the $\mathrm{H}_{3} \mathrm{O}^{+}$ column density in the QC is estimated from the model shown in Fig. 8. With $N\left(\mathrm{H}_{3} \mathrm{O}^{+}\right)=8.4 \times 10^{16} \mathrm{~cm}^{-2}$, i.e. just the same column as used for $\mathrm{H}_{2} \mathrm{O}^{+}$in Fig. 7, the model accounts for some hints of absorption in Fig. 8a-b, and is also compatible with the undetected lines shown in Fig. 8c-d. No significant upper limits on the $\mathrm{H}_{3} \mathrm{O}^{+}$column density in the outflowing components could be estimated from the absence of line wings.

\subsection{Chemical models}

In the previous sections, radiative transfer models were applied to the O-bearing molecular ions, enabling us to estimate the column densities and abundances of each species in the three kinematic components that are needed to account for the observed spectra (Table 4). Here we use the results for the abundances, inferred from the strengths of the absorption lines, as inputs to compare with chemical models, with the 
Table 4. Physical parameters for the three model components of Mrk 231

\begin{tabular}{|c|c|c|c|c|c|c|c|c|c|c|}
\hline Component & $\begin{array}{l}T_{\text {dust }}{ }^{\mathrm{a}} \\
(\mathrm{K})\end{array}$ & $\tau_{100^{\mathrm{b}}}$ & $\begin{array}{c}N(\mathrm{OH})^{\mathrm{c}} \\
\left(10^{16} \mathrm{~cm}^{-2}\right)\end{array}$ & $\begin{array}{c}N\left(\mathrm{OH}^{+}\right) \\
\left(10^{16} \mathrm{~cm}^{-2}\right)\end{array}$ & $\begin{array}{c}N\left(\mathrm{H}_{2} \mathrm{O}^{+}\right) \\
\left(10^{16} \mathrm{~cm}^{-2}\right)\end{array}$ & $\begin{array}{c}N\left(\mathrm{H}_{3} \mathrm{O}^{+}\right)^{\mathrm{d}} \\
\left(10^{16} \mathrm{~cm}^{-2}\right)\end{array}$ & $X(\mathrm{OH})^{\mathrm{e}}$ & $X\left(\mathrm{OH}^{+}\right)$ & $X\left(\mathrm{H}_{2} \mathrm{O}^{+}\right)$ & $X\left(\mathrm{H}_{3} \mathrm{O}^{+}\right)$ \\
\hline QC & 90 & 3.0 & 675 & 34 & $4.2-8.4$ & \multirow[t]{3}{*}{$\lesssim 8.4$} & $1.7 \times 10^{-6}$ & $8.7 \times 10^{-8}$ & $(1-2) \times 10^{-8}$ & \multirow[t]{3}{*}{$\lesssim 2 \times 10^{-8}$} \\
\hline HVC & 90 & 3.0 & 30 & 3 & $\lesssim 0.3$ & & $2.5 \times 10^{-6}$ & $2.5 \times 10^{-7}$ & $\lesssim 2.5 \times 10^{-8}$ & \\
\hline LEC & 55 & 0.5 & 5 & $\sim 1$ & $\lesssim 0.05$ & & $2.5 \times 10^{-6}$ & $\sim 5 \times 10^{-7}$ & $\lesssim 2.5 \times 10^{-8}$ & \\
\hline
\end{tabular}

a Dust temperature associated with the continuum source against which the molecular absorption is produced.

${ }^{b}$ Continuum optical depth of the continuum source against which the molecular absorption is produced. In the QC, it coincides with the region where the absorption is produced, but in the outflowing components (HVC and LEC) corresponds to the background continuum source (see Fig. 12 in GA17).

c From GA17.

${ }^{\mathrm{d}}$ No significant upper limits in the outflowing components.

e The molecular abundances in the QC are inferred by assuming a gas-to-dust ratio of 100 and a mass absorption coefficient of $k_{\mathrm{abs}}=44.5$ $\mathrm{cm}^{2} / \mathrm{g}$ of dust at $100 \mu \mathrm{m}$ (González-Alfonso et al. 2014b). For the outflowing components, an $\mathrm{OH}$ abundance of $2.5 \times 10^{-6}$ relative to $\mathrm{H}$ nuclei is adopted (GA17), and all other abundances are derived from this normalization.

goal of estimating the ionization rate $\zeta$ of the molecular gas components.

The chemical models presented here are the same as in GA13, which were performed with the chemical code described in detail by Bruderer et al. (2009), based on previous work by Stäuber et al. (2005) and Doty et al. (2002). Briefly, the chemical models calculate the steady state abundances of all relevant species based on the UMIST06 reaction rates (Woodall et al. 2007). The gas with $\mathrm{H}$ nuclei density $n_{\mathrm{H}}$ and molecular fraction $f_{\mathrm{H}_{2}}$ is directly exposed to a cosmic ray and/or X-ray flux that produces a total ionization rate per $\mathrm{H}$ nucleus $\zeta$ (including secondary ionizations). We ignore any other external agent (e.g. dissociating UV radiation, though internally generated UV radiation is included) and also the effect of negatively charged PAHs (Hollenbach et al. 2012). Model results basically depend on $\zeta / n_{\mathrm{H}}$, the molecular fraction $f_{\mathrm{H}_{2}}$, the gas temperature $\left(T_{\text {gas }}\right)$, and the gas-phase oxygen abundance $(X(\mathrm{O}))$. We have generated a grid of models with representative values of $T_{\text {gas }}=150,550$, and $1000 \mathrm{~K}$, solar metallicities with $X(\mathrm{O})=3 \times 10^{-4}$, and $\zeta / n_{\mathrm{H}}$ in the range $5 \times 10^{-19}-5 \times 10^{-16} \mathrm{~cm}^{3} \mathrm{~s}^{-1}$. Because of uncertainties regarding $\mathrm{H}_{2}$ formation on warm dust grains, and the lack of equilibrium in the $\mathrm{H}_{2}$ fraction that may affect mostly the outflow components (Richings \& Faucher-Giguère 2018) but also the QC, $f_{\mathrm{H}_{2}}$ is considered a free parameter.

\subsubsection{The $Q C$}

The predicted chemical model abundances of $\mathrm{OH}^{+}, \mathrm{OH}$, $\mathrm{H}_{2} \mathrm{O}^{+}$, and $\mathrm{H}_{3} \mathrm{O}^{+}$and their ratios are plotted in Fig. 9 as a function of $\zeta / n_{\mathrm{H}}$ and compared with the ranges allowed by the model fits to the observations of the QC. The most reliable observational values are the abundance ratios shown in the left-hand panels, for which an uncertainty of a factor 1.5 is adopted. Because $\mathrm{H}_{3} \mathrm{O}^{+}$is not clearly detected in Mrk 231, we adopt a large range of $4-40$ for the $\mathrm{OH}^{+} / \mathrm{H}_{3} \mathrm{O}^{+}$ratio. The absolute abundances are shown in the right-hand panels, for which a higher uncertainty, of a factor of 2 , is adopted. Chemical models are shown for $T_{\text {gas }}=150$ and $550 \mathrm{~K}$, and for two fiducial values of the molecular fraction, $f_{\mathrm{H}_{2}}=0.25$ and 0.50 .

Inspection of Fig. 9 highlights the unique role of $\mathrm{OH}^{+}$ in probing the ionization rate of the molecular gas, as it is the only species for which the abundance increases monotonically with $\zeta / n_{\mathrm{H}}$ up to the most extreme values (for fixed $T_{\text {gas }}$ and $f_{\mathrm{H}_{2}}$ ). By contrast, the abundances of all other considered species decline with increasing $\zeta / n_{\mathrm{H}}$ above several $\times 10^{-17} \mathrm{~cm}^{3} \mathrm{~s}^{-1}$. In addition, Fig. 9 indicates that results for both the absolute abundances and abundance ratios are sensitive to both $f_{\mathrm{H}_{2}}$ and $T_{\text {gas }}$. Once $\mathrm{OH}^{+}$is formed, it can be destroyed either by generating $\mathrm{H}_{2} \mathrm{O}^{+}$, or through the competing dissociative recombination that breaks the pathway to generate $\mathrm{H}_{2} \mathrm{O}^{+}$and $\mathrm{H}_{3} \mathrm{O}^{+}$. Increasing $T_{\text {gas }}$, the recombination rate decreases and the channel that produces $\mathrm{H}_{2} \mathrm{O}^{+}$and $\mathrm{H}_{3} \mathrm{O}^{+}$ (i.e. $\mathrm{OH}^{+}+\mathrm{H}_{2} \rightarrow \mathrm{H}_{2} \mathrm{O}^{+}+\mathrm{H}$ and $\mathrm{H}_{2} \mathrm{O}^{+}+\mathrm{H}_{2} \rightarrow \mathrm{H}_{3} \mathrm{O}^{+}+\mathrm{H}$ ) is reinforced, lowering the $\mathrm{OH}^{+} / \mathrm{H}_{2} \mathrm{O}^{+}$and $\mathrm{OH}^{+} / \mathrm{H}_{3} \mathrm{O}^{+}$ratios. In addition, the $\mathrm{OH}$ abundance is boosted at high $T_{\text {gas }}$ mainly because the activation barrier of $\mathrm{O}+\mathrm{H}_{2} \rightarrow \mathrm{OH}+\mathrm{H}$ is overcome (e.g. Elitzur \& de Jong 1978), thus increasing the $\mathrm{OH} / \mathrm{OH}^{+}$ratio. On the other hand, the abundance of $\mathrm{OH}^{+}$relative to the other species also decreases with increasing $f_{\mathrm{H}_{2}}$, because the above reactions that generate $\mathrm{H}_{2} \mathrm{O}^{+}$and $\mathrm{H}_{3} \mathrm{O}^{+}$ proceed more efficiently. Therefore, to explain the inferred abundance ratios (shaded regions in Fig. 9), higher values of $\zeta / n_{\mathrm{H}}$ are required if higher values for $f_{\mathrm{H}_{2}}$ and $T_{\text {gas }}$ are adopted. 

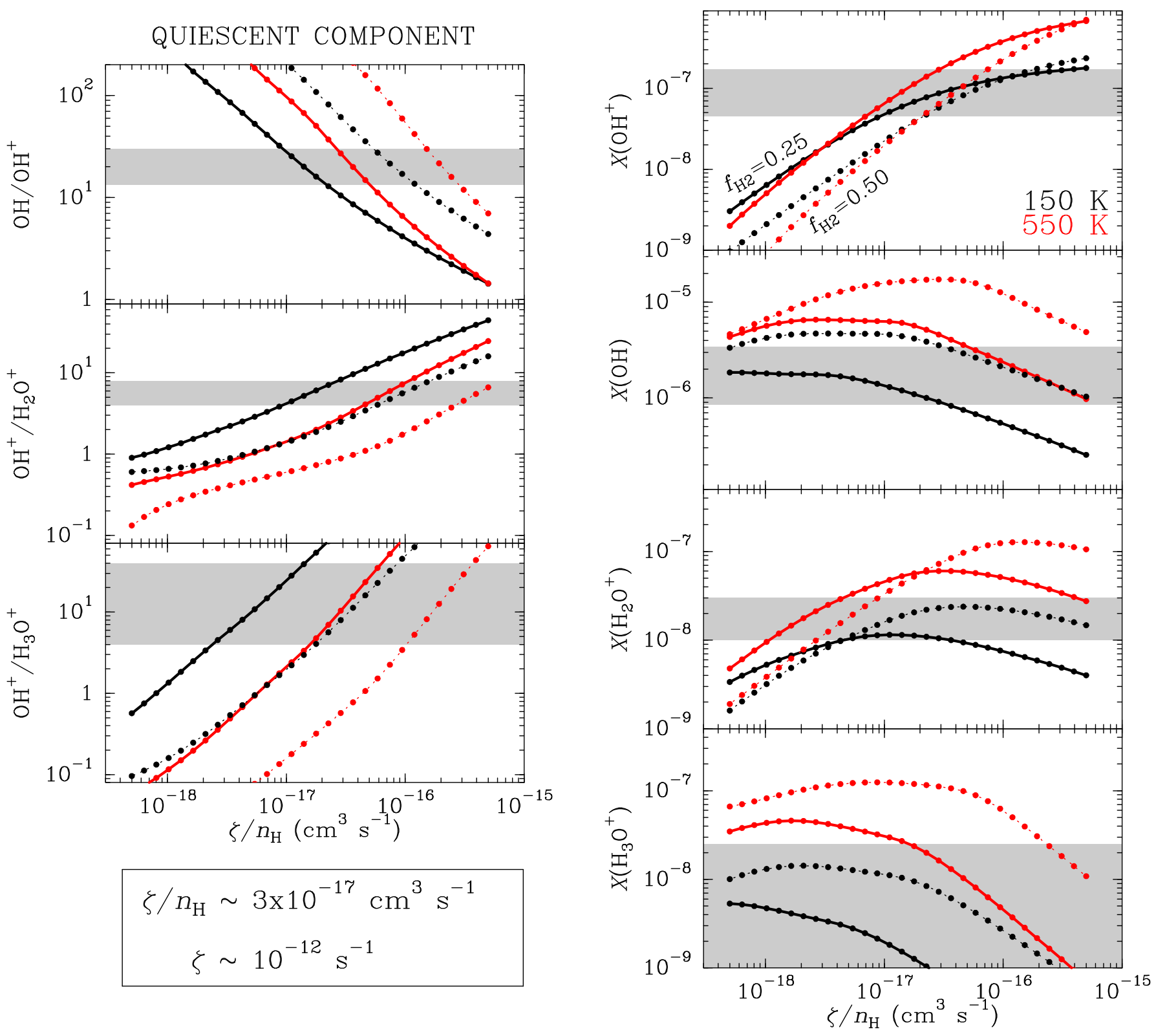

Figure 9. Chemical models (from GA13) for the QC. Left panels: the predicted abundance ratios $\mathrm{OH} / \mathrm{OH}^{+}, \mathrm{OH}^{+} / \mathrm{H}_{2} \mathrm{O}^{+}$, and $\mathrm{OH}^{+} / \mathrm{H}_{3} \mathrm{O}^{+}$, as a function of $\zeta / n_{\mathrm{H}}$. Right panels: the absolute abundances of the considered molecular species. Black and red lines indicate results for $T_{\mathrm{gas}}=150$ and $550 \mathrm{~K}$, respectively, while thick-solid and dotted lines use molecular fractions of $f_{\mathrm{H}_{2}}=0.25$ and 0.50 , respectively. The horizontal shaded regions indicate the range of values favored by Herschel observations and our radiative transfer models for the QC. Our favored solution is in the range between the thick curves $\left(T_{\text {gas }}=150-550 \mathrm{~K}, f_{\mathrm{H}_{2}}=0.25\right)$, for which $\zeta / n_{\mathrm{H}} \sim 3 \times 10^{-17} \mathrm{~cm}^{3} \mathrm{~s}^{-1}$ (bottom left).

A range of physical conditions, and in particular in density, $T_{\text {gas }}, f_{\mathrm{H}_{2}}$, and $\zeta / n_{\mathrm{H}}$, will be naturally present in the $\mathrm{QC}$, though densities of several $\times 10^{4} \mathrm{~cm}^{-3}$ are most likely prevalent (Mashian et al. 2015). At densities $\gtrsim 5 \times 10^{4} \mathrm{~cm}^{-3}$, the gas cools efficiently and thermal balance (including only cosmic ray heating) indicates that $T_{\text {gas }}$ will be moderate even for high $\zeta \sim 5 \times 10^{-13} \mathrm{~s}^{-1}(<200 \mathrm{~K}$; Papadopoulos et al. 2011, 2014). Observationally, the CO lines from $J_{\text {up }}=5$ to 11 are fitted in Mrk 231 with $T_{\text {gas }} \approx 300 \mathrm{~K}$ (Mashian et al. 2015). We thus expect $T_{\text {gas }}$ in the range between the 150 and 550
K cases shown in Fig. 9, but probably closer to the low- $T_{\text {gas }}$ solution.

To account for the inferred abundances and ratios, the required value of $\zeta / n_{\mathrm{H}}$ increases by a factor of $\sim 5$ if $f_{\mathrm{H}_{2}}$ is increased from 0.25 to 0.50 (Fig. 9). Nevertheless, the combination of very warm $T_{\text {gas }}=550 \mathrm{~K}$ and $f_{\mathrm{H}_{2}} \gtrsim 0.50$ is ruled out, as it grossly overpredicts the abundances of $\mathrm{OH}, \mathrm{H}_{2} \mathrm{O}^{+}$, and $\mathrm{H}_{3} \mathrm{O}^{+}$unless an extremely high $\zeta / n_{\mathrm{H}}>10^{-16} \mathrm{~cm}^{3} \mathrm{~s}^{-1}$ is assumed, but this would be hardly compatible with a high molecular fraction (GA13). Indeed, the $f_{\mathrm{H}_{2}}=0.50$ case is 
only compatible with $T_{\text {gas }}=150 \mathrm{~K}$, as it gives $\zeta / n_{\mathrm{H}} \gtrsim 5 \times$ $10^{-17} \mathrm{~cm}^{3} \mathrm{~s}^{-1}$ that is still consistent with a maximum $f_{\mathrm{H}_{2}}^{\max } \sim$ 0.5 (GA13). On the other side, all molecular abundances start to decline strongly at $f_{\mathrm{H}_{2}}<15 \%$ (not shown), and the molecular chemistry is simply suppressed. Our favored solution is therefore $T_{\text {gas }}=150-500 \mathrm{~K}$ and $f_{\mathrm{H}_{2}}=0.15-0.5$, with decreasing $T_{\text {gas }}$ for increasing $f_{\mathrm{H}_{2}}$, for which we infer $\zeta / n_{\mathrm{H}} \sim(3 \pm 2) \times 10^{-17} \mathrm{~cm}^{3} \mathrm{~s}^{-1}$. The electron abundance is $\sim 10^{-3}$.

The quoted solution gives results compatible with the observational constraints for the $\mathrm{OH} / \mathrm{OH}^{+}, \mathrm{OH}^{+} / \mathrm{H}_{2} \mathrm{O}^{+}$, and $\mathrm{OH}^{+} / \mathrm{H}_{3} \mathrm{O}^{+}$ratios, and also nicely brackets the absolute abundances estimated for $\mathrm{OH}^{+}, \mathrm{OH}, \mathrm{H}_{2} \mathrm{O}^{+}$, and $\mathrm{H}_{3} \mathrm{O}^{+}$. In particular, the predicted range for the abundance of $\mathrm{OH}$ $\left((0.8-5) \times 10^{-6}\right)$ is consistent with the value inferred in ULIRGs from the OH $35 \mu \mathrm{m}$ transition (Stone et al. 2017). As will be shown in a future work, however, these physical conditions underestimate the $\mathrm{H}_{2} \mathrm{O}$ abundance. A range of physical values will be naturally present in the $\mathrm{QC}$, and it is quite possible that $\mathrm{H}_{2} \mathrm{O}$ preferentially forms and survives in warm, high-density regions (i.e. $\zeta / n_{\mathrm{H}}$ lower than average). The strong submillimeter line of $\mathrm{H}_{2} \mathrm{O}^{+}$could also partially arise there.

We can now evaluate the role of formation pumping, relative to collisional pumping, in the emission of strong groundstate lines of $\mathrm{OH}^{+}$. The number of photons generated per unit time and volume in the three ground-state $1_{J}-0_{1}$ lines via formation pumping is $\gamma_{\text {pump }}^{1,-0} \sim n_{\mathrm{H}} \zeta \epsilon_{\mathrm{OH}^{+}}(\mathrm{GA} 13)$, where $\epsilon_{\mathrm{OH}^{+}}$is the efficiency with which ionizations of hydrogen are transferred to $\mathrm{OH}^{+}$production (Neufeld et al. 2010). We adopt a value $\epsilon_{\mathrm{OH}^{+}}=0.5$, which is an upper limit because neutralization of $\mathrm{H}^{+}$on small grains or PAHs is expected to decrease the efficiency of $\mathrm{OH}^{+}$formation (Hollenbach et al. 2012; Indriolo et al. 2012). Ignoring excitation by the radiation field, the corresponding value generated through collisional excitation is $\gamma_{\text {col }}^{1_{1}-0_{1}} \sim f_{0_{1}} n_{\mathrm{H}}^{2} X\left(\mathrm{OH}^{+}\right) \sum_{u} C_{0_{1} \rightarrow u}$, where $f_{0_{1}}$ is the fractional population in the ground $0_{1}$ level, and the sum extends to all collisional rates from the $0_{1}$ level to any other excited one (yielding $1.55 \times 10^{-9} \mathrm{~cm}^{3} \mathrm{~s}^{-1}$ at $300 \mathrm{~K}$; Lique et al. 2016). The ratio of these quantities is

$$
\frac{\gamma_{\text {pump }}^{1,-00_{1}}}{\gamma_{\text {col }}^{1_{J}-0_{1}}} \sim \frac{\left(\zeta / n_{\mathrm{H}}\right) \epsilon_{\mathrm{OH}^{+}}}{f_{0_{1}} X\left(\mathrm{OH}^{+}\right) \sum_{u} C_{0_{1} \rightarrow u}} \sim \frac{0.1}{f_{0_{1}}} \times \frac{\zeta / n_{\mathrm{H}}}{3 \times 10^{-17} \mathrm{~cm}^{3} \mathrm{~s}^{-1}}
$$

where $X\left(\mathrm{OH}^{+}\right)=10^{-7}$ is adopted (Fig. 9). In our best-fit models, $f_{0_{1}}$ lies in the range $\sim 0.2-0.6$, and thus collisional excitation appears to dominate ${ }^{7}$.

\footnotetext{
7 We have also evaluated the expected flux arising from formation pumping in the three ground-state $\mathrm{OH}^{+}$lines using Appendix A in GA13 and including also the outflowing components, with the prediction that $\lesssim 30 \mathrm{Jy} \mathrm{km} \mathrm{s}^{-1}$ are expected, much lower than the observed fluxes (Table 3).
}

In summary, the combination of Herschel observations, radiative transfer models, and chemical models, favor $\zeta / n_{\mathrm{H}} \sim$ $(3 \pm 2) \times 10^{-17} \mathrm{~cm}^{3} \mathrm{~s}^{-1}$ in the Klöckner et al. torus. This is similar to, though likely somewhat higher than, the value inferred in the nuclear region of Arp 220 (GA13), and indeed the continuum-normalized absorption at systemic velocities in the excited $\mathrm{OH}^{+}$and $\mathrm{H}_{2} \mathrm{O}^{+}$lines are similar in both sources. One obvious difference, however, is the high densities inferred in Mrk 231 from the ground-state lines of $\mathrm{OH}^{+}, n_{\mathrm{H}} \sim 2 \times 10^{5} \mathrm{~cm}^{-3}$ (\$3.1.1), pointing to a very high value of $\zeta$ in the nuclear region. The caveat, however, is that the column densities and abundances are primarily derived from the (excited) absorption lines that are formed in front of the continuum source, while the above density is inferred from the (ground-state) emission lines, which do not have the above requirement and are primarily formed in a different region of the torus. In fact, while the peak absorption of the excited $\mathrm{OH}^{+} 2_{J}-1_{J^{\prime}}$ lines is blueshifted, the peak emission of the ground-state $\mathrm{OH}^{+} 1_{J}-0_{J^{\prime}}$ lines is redshifted (Fig. 5), unambiguosly indicating distinct formation regions for the absorption and emission lines. Observations of $\mathrm{CO}$ $2-1$ and 3-2 show that the redshifted line wing emission is stronger than that of the blueshifted wing (Feruglio et al. 2015). Therefore, the density in the environments where the absorption lines are generated may be different, and probably lower than estimated from the emission lines. Nevertheless, the warm CO SLED observed in the source indicates that the overall density in the nuclear region of Mrk 231 is at least several $\times 10^{4} \mathrm{~cm}^{-3}$ (van der Werf et al. 2010; Mashian et al. 2015), from which we conservatively estimate $\zeta \sim 10^{-12} \mathrm{~s}^{-1}$ with an uncertainty of up to a factor $\sim 3$. This value is still $>10^{3}$ times the mean value found in the Milky Way (Indriolo et al. 2015), $>10$ times the value estimated in a spiral galaxy at $z=0.89$ (Muller et al. 2016), and $\gtrsim 10$ times the maximum values found in the Galactic center by Indriolo et al. (2015).

\subsubsection{The outflowing components}

Because of the limited observational constraints for the outflowing gas, we model the HVC and the LEC with a single chemical model and consider only the abundances of $\mathrm{OH}, \mathrm{OH}^{+}$, and $\mathrm{H}_{2} \mathrm{O}^{+}$(Fig. 10). In the outflow, a very low $\mathrm{OH} / \mathrm{OH}^{+} \sim 10$ abundance ratio is inferred (Table 4), which points to values of $\zeta / n_{\mathrm{H}}$ even higher than in the QC.

To constrain $f_{\mathrm{H}_{2}}$ and the density $n_{\mathrm{H}}$, we follow Richings \& Faucher-Giguère $(2018,2017)$ to characterize the physical conditions in the high-velocity (partially) molecular outflows driven by luminous AGN. In their thermo-chemical 3D models, the ISM gas is swept out in a forward shock driven by a hot shocked bubble, attaining high $T_{\text {gas }} \sim 10^{7} \mathrm{~K}$ where all molecules are destroyed. The molecular gas reforms downstream, and one key result of these models is that there is a range in the post-shock density $\left(n_{\mathrm{H}}\right.$, hence also in the pre- 

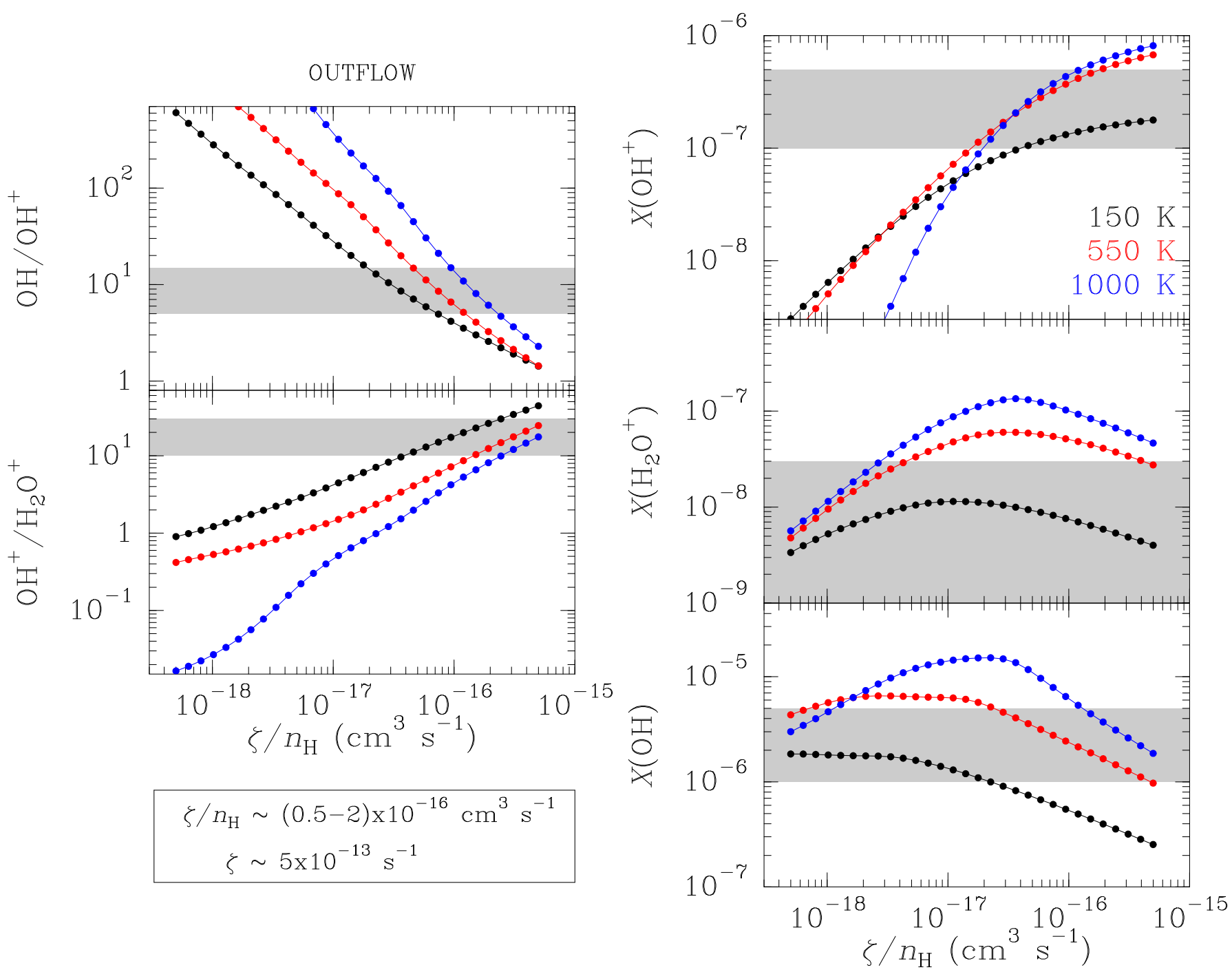

Figure 10. As Fig. 9, but for the outflowing components. Left panels: the predicted abundance ratios $\mathrm{OH} / \mathrm{OH}^{+}$and $\mathrm{OH}^{+} / \mathrm{H}_{2} \mathrm{O}^{+}$as a function of $\zeta / n_{\mathrm{H}}$. Right panels: the absolute abundances $\mathrm{OH}, \mathrm{OH}^{+}$, and $\mathrm{H}_{2} \mathrm{O}^{+}$. Model predictions are shown for $T_{\text {gas }}=150$ (in black), 550 (red), and $1000 \mathrm{~K}$ (blue), and the molecular fraction is $f_{\mathrm{H}_{2}}=0.25$ in all models. At the bottom left corner, the favored values for both $\zeta / n_{\mathrm{H}}$ and $\zeta$ for the outflow are indicated.

shock density $n_{\mathrm{H} 0}$ ) that allows for high-velocities of the outflowing molecular gas: if $n_{\mathrm{H}}$ is too low, the post-shock gas cannot cool in time to form molecules, while if $n_{\mathrm{H} 0}$ is too high, the post-shock gas does not approach the observed high velocities. Values of $n_{\mathrm{H} 0}$ around $10 \mathrm{~cm}^{-3}$ and post-shock gas densities around afew $\times 10^{3}-10^{4} \mathrm{~cm}^{-3}$, appear to be an appropriate compromise between quick enough $\mathrm{H}_{2}$ formation and high outflowing velocities (Richings \& Faucher-Giguère 2017), and these are indeed the typical densities we found in our best fit model for the HVC (GA17). In addition, the conversion to molecular gas is far from complete, with $f_{\mathrm{H}_{2}} \sim 0.25$ being favored for high velocity outflows (Richings \& Faucher-Giguère 2018).

Chemical models for the abundances and ratios of $\mathrm{OH}$, $\mathrm{OH}^{+}$, and $\mathrm{H}_{2} \mathrm{O}^{+}$in Fig. 10 therefore use $f_{\mathrm{H}_{2}} \sim 0.25$ and, because the gas is very warm at the "knee" of the $\mathrm{H}_{2}$ forma- tion, models for $T_{\text {gas }}=1000 \mathrm{~K}$ are also considered. Results for the abundance ratios are then consistent with $\zeta / n_{\mathrm{H}} \sim$ $(0.5-2) \times 10^{-16} \mathrm{~cm}^{3} \mathrm{~s}^{-1}$. Papadopoulos et al. (2011) show that for an ionization rate of $5 \times 10^{-13} \mathrm{~s}^{-1}$ (due to cosmic rays, see $\S 4.2$ ) and gas densities of $5 \times 10^{3} \mathrm{~cm}^{-3}, T_{\text {gas }}$ is well above $200 \mathrm{~K}$ even at high visual extinctions, and this is a lower limit because of additional heating sources in the post-shock gas. Therefore, the high $T_{\text {gas }}=550-1000 \mathrm{~K}$ solutions are favored for the outflowing gas, which yield $\zeta / n_{\mathrm{H}} \sim(1-2) \times 10^{-16} \mathrm{~cm}^{3} \mathrm{~s}^{-1}$ and $\zeta \sim 5 \times 10^{-13} \mathrm{~s}^{-1}$. This solution yields absolute abundances of $\mathrm{OH}^{+}$and $\mathrm{H}_{2} \mathrm{O}^{+}$above the observationally determined ranges, probably meaning that only a fraction of the outflowing column of gas is subject to the above extreme conditions. For the HVC, where $N\left(\mathrm{OH}^{+}\right) \sim 3 \times 10^{16} \mathrm{~cm}^{-2}$ (Table 4), the corresponding hydrogen column is $4.3 \times 10^{22} \mathrm{~cm}^{-2}$ for $X\left(\mathrm{OH}^{+}\right) \sim 7 \times 10^{-7}$, 
i.e. less than half the total column of the HVC component. As the molecular gas cools and its density increases (with the concomitant decrease of $\zeta / n_{\mathrm{H}}$ ), the $\mathrm{OH}^{+}$abundance is expected to decrease (Fig. 10) and thus we expect that $\mathrm{OH}^{+}$ primarily probes the most extreme, partially molecular environments associated with high $T_{\text {gas }}$ and $\zeta / n_{\mathrm{H}}$-including the molecular formation region of the post-shock gas.

One caveat to the favored solution concerns the shallow H I $21 \mathrm{~cm}$ absorption found by Morganti et al. (2016) in the outflow of Mrk 231. For $f_{\mathrm{H}_{2}}=0.25$, we expect a column $N(\mathrm{H} \mathrm{I}) \sim 3.2 \times 10^{22} \mathrm{~cm}^{-2}$ in the HVC, and about half of this column is at blueshifted velocities $|v|>500 \mathrm{~km} \mathrm{~s}^{-1}$. While this is still consistent with the observed $\mathrm{H} \mathrm{I} 21 \mathrm{~cm}$ absorption at blueshifted velocities if $T_{\text {spin }} \gtrsim 1000 \mathrm{~K}$, the model would overestimate the observed absorption if $T_{\text {spin }}$ were significantly lower. On the other hand, at the expected densities $>10^{3} \mathrm{~cm}^{-3}$ the $\mathrm{H} \mathrm{I} 21 \mathrm{~cm}$ line is thermalized and $T_{\text {spin }}=T_{\text {gas }}$ (Morganti et al. 2016). Therefore, the $\mathrm{OH}^{+}$and $\mathrm{H}$ I $21 \mathrm{~cm} \mathrm{ab}-$ sorption at high blueshifted velocities are expected to arise in the same outflowing component as long as $T_{\text {gas }}$ is high. Nevertheless, the shallow H I absorption may be better explained if a significant fraction of the synchrotron $21 \mathrm{~cm}$ continuum is generated in the forward shock (Faucher-Giguère \& Quataert 2012; Nims et al. 2015; Liu et al. 2017, see also §4.3), i.e. ahead of the neutral and molecular outflowing gas, in such a way that only a fraction of the radio continuum is background relative to H I (see Figs. 1 in Faucher-Giguère \& Quataert 2012; Zubovas \& King 2014; Richings \& Faucher-Giguère 2017).

\section{DISCUSSION}

\subsection{Summary of radiative transfer and chemical models}

A picture of both the torus around the AGN of Mrk 231 and the outflowing molecular gas emerges from the combination of Herschel spectroscopic observations in the far-IR, radiative transfer models to infer the molecular column densities and abundances, and chemical models that enable an estimation of the physical parameters responsible for the observed chemistry.

For the torus (denoted as QC above because it is responsible for the relatively narrow spectral features observed near systemic velocities), we find high column densities of dust, $\mathrm{OH}$ and $\mathrm{OH}^{+}$, and relatively high $\mathrm{OH}^{+} / \mathrm{H}_{2} \mathrm{O}^{+} \sim 4-8$ and $\mathrm{OH}^{+} / \mathrm{H}_{3} \mathrm{O}^{+}>4$ ratios. The densities are high, but molecular fractions $f_{\mathrm{H}_{2}} \lesssim 50 \%$ are inferred together with high ionization rates $\left(\zeta \sim 10^{-12} \mathrm{~s}^{-1}\right)$. Due to the extreme conditions around the AGN, which is expected to produce high quantities of cosmic rays, only molecular gas at high densities, lowering the $\zeta / n_{\mathrm{H}}$ ratio, can survive. All gas with densities below a few $\times 10^{4} \mathrm{~cm}^{-3}$ will be ionized and ultimately dispersed.
The molecular outflow observed in $\mathrm{OH}$ shows, on the other hand, even higher $\mathrm{OH}^{+}$columns relative to both $\mathrm{OH}$ and $\mathrm{H}_{2} \mathrm{O}^{+}$. This appears to point to even higher values of $\zeta / n_{\mathrm{H}} \sim 10^{-16} \mathrm{~cm}^{3} \mathrm{~s}^{-1}$, most probably due to densities lower than in the QC, and low molecular fractions. Under these conditions the $\mathrm{C}^{+}$abundance is high $\left(\gtrsim 10^{-4}\right)$, which may explain the approximately 1:1 relationship in the gas masses derived from $\mathrm{OH}$ and [C II] (Janssen et al. 2016). Nevertheless, a range of conditions is expected because efficient formation of $\mathrm{CO}$ and $\mathrm{HCN}$ requires lower $\zeta / n_{\mathrm{H}}$ and higher $f_{\mathrm{H}_{2}}$. Indeed, evidence for chemical differentiation has been found in the molecular outflow of Mrk 231 (Lindberg et al. 2016).

Quantitatively, the main caveat to our analysis concerns the absolute molecular abundances in the outflow, for which we have adopted a reference, fiducial value for $\mathrm{OH}$ of $2.5 \times 10^{-6}$ (GA17) -similar to that inferred at systemic velocities from several transitions by assuming a gas-to-dust ratio of $\sim 100$ by mass (González-Alfonso et al. 2012; Stone et al. 2017). We emphasize that the adopted $\mathrm{OH}$ abundance is relative to $\mathrm{H}$ nuclei in the outflow, regardless of whether it is in atomic or molecular form (Richings \& Faucher-Giguère 2018). In this way, we argue from the present study that the abundances of $\mathrm{OH}$ and $\mathrm{OH}^{+}$adopted for the outflow are most probably accurate to within a factor $\sim 2.5$. If $X(\mathrm{OH})$ were much higher than $2.5 \times 10^{-6}, X\left(\mathrm{OH}^{+}\right)$would also be much higher than $2.5 \times 10^{-7}$, which would be hard to account for with current chemical models. On the other side, if $X(\mathrm{OH})$ were much lower than $2.5 \times 10^{-6}$, the energetics associated with the outflow (which scale as $X(\mathrm{OH})^{-1}$ ) would be very hard to reconcile with detailed feedback models (Richings \& FaucherGiguère 2018, 2017).

\subsection{A giant Cosmic Ray Dominated Region in the nucleus of Mrk 231}

What mechanism is responsible for the high ionization rates that we have derived? NUSTAR observations of Mrk 231 have revealed an intrinsically weak source of X-rays, with a luminosity $L_{X}(2-10 \mathrm{keV})=4 \times 10^{42} \mathrm{erg} \mathrm{s}^{-1}$ (Teng et al. 2014). Assuming that the $X$-rays originate in the AGN, the unattenuated X-ray flux at a distance of $r_{\mathrm{pc}} \mathrm{pc}$ is $F_{X}(2-$ $10 \mathrm{keV})=3.3 \times\left(r_{\mathrm{pc}} / 100\right)^{-2} \mathrm{erg} \mathrm{s}^{-1} \mathrm{~cm}^{-2}$. Following Maloney et al. (1996), the ionization rate produced by the X-rays is given by $\zeta\left(\mathrm{s}^{-1}\right) \sim 10^{-13} F_{X} / N_{22}^{0.9}$, where $N_{22}=N / 10^{22} \mathrm{~cm}^{-2}$ and $N$ is the hydrogen column density attenuating the $\mathrm{X}$-ray flux. From these estimates, and given the extremely high values of $\zeta \sim 5 \times 10^{-13} \mathrm{~s}^{-1}$ we infer at $\gtrsim 100 \mathrm{pc}$ and affecting columns of gas $\gtrsim 2 \times 10^{22} \mathrm{~cm}^{-2}$ (Table 2 in GA17), we find that the X-rays from the AGN are probably unable to account for the Herschel/PACS observations of molecular ions in Mrk 231. Even if a putative luminous X-ray source were located at or close to the location of the AGN and strongly 

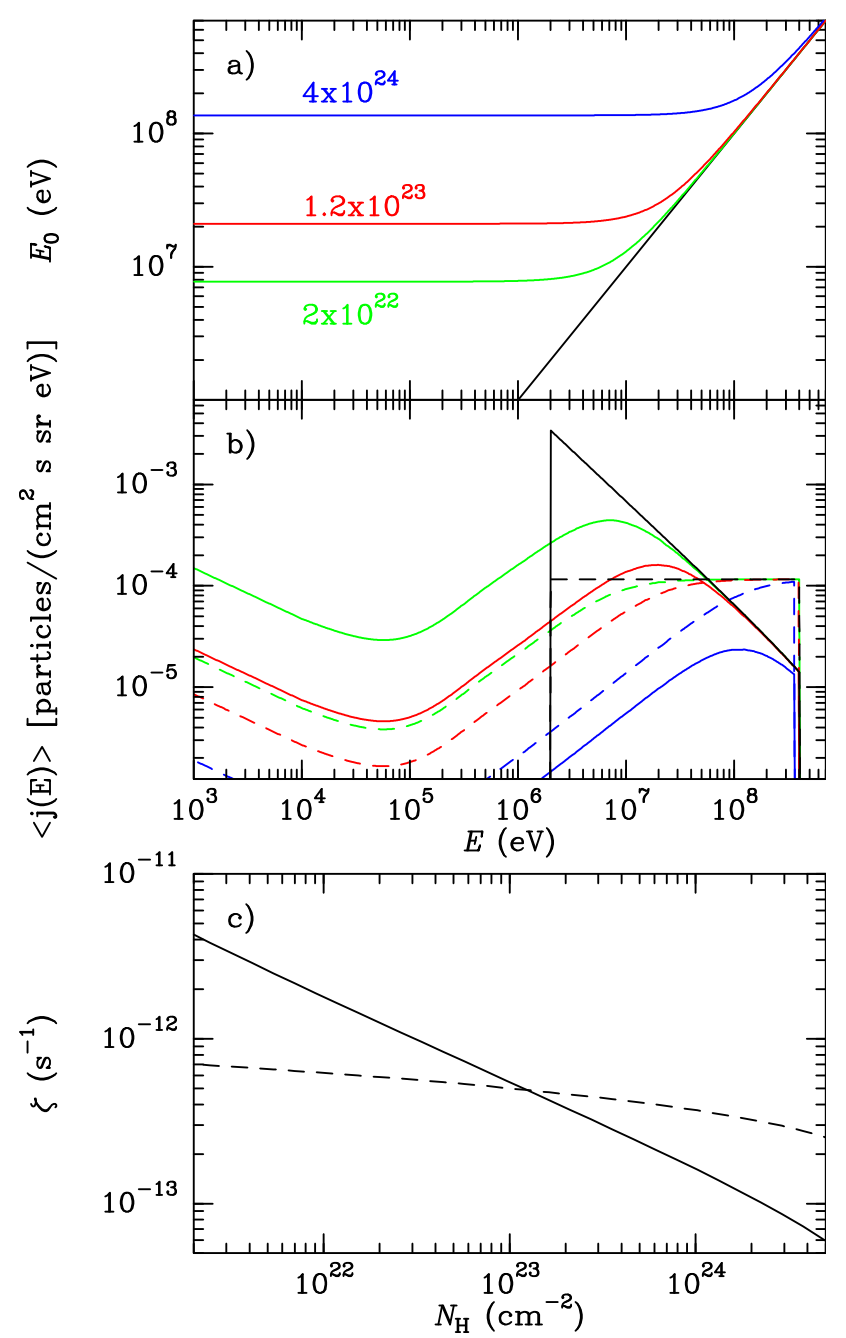

Figure 11. a) The CR proton energy $E$ after crossing a column $N_{\mathrm{H}}$ in terms of the incident CR energy at the cloud surface $E_{0}$ (eq. 2). Columns of $N_{\mathrm{H}}=2 \times 10^{22}, 1.2 \times 10^{23}$, and $4 \times 10^{24} \mathrm{~cm}^{-2}$, corresponding to those inferred for the LEC, HVC, and QC components, are considered. There are thresholds in $E_{0}$ of 8, 21, and 140 $\mathrm{MeV}$, respectively, which are the minimum incident $\mathrm{CR}$ energies that can penetrate the corresponding columns. The black line indicates $E_{0}=E$. b) The black lines show our adopted angle-averaged incident $\mathrm{CR}$ proton spectra (i.e. $\left\langle j_{0}\left(E_{0}\right)>\right.$ ). We have used a powerlaw spectrum (solid line), with $<j_{0}>\propto p^{-2}$, and a flat distribution (dashed line). The colored curves show the propagated spectra $\left.\left(<j\left(E, N_{\mathrm{H}}\right)\right\rangle\right)$ after crossing the three columns of gas considered in a) (solid and dashed lines for the power-law and flat distributions, respectively). c) The inferred ionization rate at the center of a spherical cloud as a function of its column density, for both the power-law (solid) and flat (dashed) incident spectra.

attenuated by foreground gas, the excited lines of $\mathrm{OH}^{+}$are observed in absorption against a continuum that is optically thick even in the far-IR, which would protect the outflowing gas from strong X-ray ionization. An alternative is that the observed $\mathrm{X}$-ray emission originates in the forward shock (Nims et al. 2015), in which case the source of X-rays would be more spatially linked to the molecular outflow, although the mentioned X-ray attenuation would still apply. We thus resort in the following to the potential role played by CRs.

An ultra-fast outflow (UFO) with a velocity of $\sim 2 \times 10^{4}$ $\mathrm{km} \mathrm{s}^{-1}$ has been recently identified in the Chandra and $N u S$ TAR X-ray data of Mrk 231 (Feruglio et al. 2015). The authors estimate that the UFO covering fraction is consistent with unity, with mass outflow and energy rates in the range $\dot{M}_{\mathrm{UFO}}=0.3-2.1 \mathrm{M}_{\odot} \mathrm{yr}^{-1}$ and $\dot{E}_{\mathrm{UFO}}=(0.38-2.7) \times 10^{44}$ $\mathrm{erg} \mathrm{s}^{-1}$, respectively. The wind mechanical power is significantly lower than that inferred in IRAS F11119+3257, where a molecular outflow is also detected (Tombesi et al. 2015; Veilleux et al. 2017). The UFO in Mrk 231 is detected during an epoch of low radio emission (i.e. suppressed jet activity) but undetected in epochs of high radio emission, suggesting high variablity and possible jet-wind mutual exclusion (Reynolds et al. 2017). The UFO, jet activity (e.g. Ulvestad et al. 1999), BAL wind (e.g. Veilleux et al. 2013b, 2016), and molecular outflow suggest the accompanying generation of CRs (Faucher-Giguère \& Quataert 2012; Nims et al. 2015; Liu et al. 2017) that can permeate and ionize the molecular gas.

We attempt here to evaluate the energetic requirements of the CR field in Mrk 231 that is responsible for the inferred ionization rates. The energy loss of a CR proton that has propagated through an atomic cloud with column $N_{\mathrm{H}}=2 \times$ $10^{22}, 1.2 \times 10^{23}$, and $4 \times 10^{24} \mathrm{~cm}^{-2}$ is shown in Fig. 11a. We have used the continuous-slowing-down-approximation (Padovani et al. 2009) and have calculated $E_{0}$ numerically for a given final energy $E$ according to

$$
\int_{E}^{E_{0}} \frac{d E}{L_{k}(E)}=N_{\mathrm{H}}
$$

where $L_{k}(E)=-d E / d N_{\mathrm{H}}$ is the energy loss function taken from the NIST database ${ }^{8}$. Figure 11a indicates that incident proton energies $E_{0}>8,21$, and $140 \mathrm{MeV}$ are required to penetrate the quoted column densities. These energies are significantly higher than the $2 \mathrm{MeV}$ protons associated with the UFO, and we thus require a different particle (CR) field that can penetrate the high columns of gas associated with the kinematic components in Mrk 231 (QC, HVC, LEC).

Given the threshold energies of Fig. 11a, and the decreasing Bethe cross section for primary ionization of atomic hydrogen $\left(\sigma_{\text {ion }} \sim E^{-0.88}\right.$ between 2 and $\left.100 \mathrm{MeV}\right)$, the ionization is expected to be mainly caused by CRs with incident energies between $E_{0} \sim 8$ and several $\times 100 \mathrm{MeV}$. Since we aim to check the ionization rate as a function of column density, we have considered CR protons with energies between

\footnotetext{
8 https://www.nist.gov/pml/stopping-power-range-tables-electronsprotons-and-helium-ions. We ignore the energy loss by pion production, relevant at proton energies above $1 \mathrm{GeV}$.
} 
2 and $400 \mathrm{MeV}$, and adopted two incident spectra: a powerlaw distribution with $\sim p^{-2}$ (solid black line in Fig. 11b), and a flat distribution (dashed black line). The normalization of each distribution is a free parameter that we vary in order to obtain appropriate values of $\zeta$. The propagated spectra after crossing the characteristic column densities quoted above are also shown in Fig. 11b with colored lines. We have again used the method outlined by Padovani et al. (2009), i.e.

$$
j\left(E, N_{\mathrm{H}}\right)=j_{0}\left(E_{0}\right) \times \frac{L_{k}\left(E_{0}\right)}{L_{k}(E)},
$$

where $j_{0}\left(E_{0}\right)$ is the incident spectrum and $j\left(E, N_{\mathrm{H}}\right)$ is the propagated spectrum after crossing a column density of $N_{\mathrm{H}}$. The propagated spectra have a minimum at $\sim 70 \mathrm{keV}$ because there is a maximum in $L_{k}(E)$ at this energy (Padovani et al. 2009).

The ionization rates at the cloud center, shown in Fig. 11c as a function of $N_{\mathrm{H}}$, are calculated as (e.g. Spitzer \& Tomasko 1968)

$$
\zeta=\frac{5}{3}\left(1+G_{10}\right) 4 \pi \int d E<j\left(E, N_{\mathrm{H}}\right)>\sigma(E)
$$

where $\sigma$ is the Bethe ionization cross section, the factor $5 / 3$ accounts for secondary ionizations, and $G_{10}=0.5$ simulates the contribution to the ionization by nuclei heavier than protons, assuming solar metallicities (see Appendix in Indriolo et al. 2009). With the normalizations showed in Fig. 11b, the values of $\zeta$ are $\sim 5 \times 10^{-13} \mathrm{~s}^{-1}$ at $N_{\mathrm{H}}=1.2 \times 10^{23} \mathrm{~cm}^{-2}$ (Fig. 11c), thus accounting for the ionization rate inferred in the outflowing HVC component. CRs with energies above $400 \mathrm{MeV}$ would be required to reproduce $\zeta \sim 10^{-12} \mathrm{~s}^{-1}$ in the QC, although CRs originating from supernovae within the QC could also provide an important contribution.

The incident angle-averaged $\mathrm{CR}$ spectrum shown in Fig. $11 \mathrm{~b}$ is related to the mass outflow rate, momentum flux, and energy flux in CRs, as

$$
\begin{gathered}
\dot{M}_{\mathrm{CR}}=(4 \pi)^{2} R^{2} f_{c} \mu m_{p} \int d E<j_{0}(E)> \\
\dot{p}_{\mathrm{CR}}=(4 \pi)^{2} R^{2} f_{c} \mu \int d E<j_{0}(E)>p(E) \\
\dot{E}_{\mathrm{CR}}=(4 \pi)^{2} R^{2} f_{c} \mu \int d E<j_{0}(E)>E,
\end{gathered}
$$

where $m_{p}$ is the proton mass, $\mu=1.4$ corrects for the mass of heavier nuclei, $p(E)$ is the proton momentum correponding to kinetic energy $E$, and $R$ is the distance to the AGN. The inclusion of the factor $f_{c}$, the covering factor of the outflow (GA17), makes our estimate conservative, as we only count the CRs incident on the molecular region traced by $\mathrm{OH}^{+}$(the aligned-momenta approach, GA17). Using $R=170$ pc and $f_{c}=0.18$, appropriate for the $\operatorname{HVC}$ (GA17), we obtain $\dot{M}_{\mathrm{CR}}=0.010-0.013 \mathrm{M}_{\odot} \mathrm{yr}^{-1}, \dot{p}_{\mathrm{CR}}=(0.6-1.6) \times 10^{34} \mathrm{dyn}$, and $\dot{E}_{\mathrm{CR}}=(0.44-1.7) \times 10^{44} \mathrm{erg} \mathrm{s}^{-1}$ for the power-law and flat distributions, respectively. Even higher values are obtained for the LEC with $R=600 \mathrm{pc}$ and $f_{c}=0.26$, but the more moderate column in this component means that protons in the high-velocity wing of the thermal distribution of the hot bubble at $\sim 10^{10} \mathrm{~K}$, as predicted by models of energy-conserving outflows (e.g. King et al. 2011; Zubovas \& King 2012; Faucher-Giguère \& Quataert 2012; Richings \& Faucher-Giguère 2018), could mix with the outflowing ISM layer and contribute significantly to increase $\zeta$. The quoted values are instantaneous (GA17, Veilleux et al. 2017), and the time-averaged values are a factor $\sim 4$ smaller.

\subsection{The generation of CRs and the forward shock in Mrk 231}

While the inferred $\dot{M}_{\mathrm{CR}} \sim 0.01 \mathrm{M}_{\odot} \mathrm{yr}^{-1}$ is significantly lower than $\dot{M}_{\mathrm{UFO}}$, it is worth noting that $\dot{E}_{\mathrm{CR}} \sim 10^{44} \mathrm{erg} \mathrm{s}^{-1}$ is similar to both $\dot{E}_{\mathrm{UFO}}$ and the time-averaged energy flux associated with the molecular outflow $\left(\sim 10^{44} \mathrm{erg} \mathrm{s}^{-1}\right.$; Feruglio et al. 2015, GA17), and equivalent to $\sim 1 \%$ of the AGN luminosity $\left(\sim 10^{46} \mathrm{erg} \mathrm{s}^{-1}\right)$. CRs are not thermalized into a hot bubble, but penetrate into the gas producing ionizations and depositing momentum as well. Although $\dot{p}_{\mathrm{CR}}$ is a lower limit as it neglects CRs magnetic mirroring (Padovani \& Galli 2011), the estimated momentum rate of the CR field still falls short of accounting for the inferred momentum rate of the molecular outflow $\left(\sim 2.5 \times 10^{36}\right.$ dyn for the HVC and LEC together, GA17).

Multitransition analysis of the $\mathrm{OH}$ doublets yields estimated sizes (radii) for the molecular outflow components in Mrk 231 of $170 \mathrm{pc}$ (HVC) and $600 \mathrm{pc}$ (LEC, GA17). These sizes are similar to those measured in the radio continuum for the inner and outer disk-like components of the source, respectively (Carilli et al. 1998; Taylor et al. 1999). It is thus tempting to relate the CR proton field responsible for the molecular ionization of the outflowing gas to the relativistic electrons that generate the disk-like synchrotron radio emission. Specifically, we evaluate whether both the CR protons and electrons can be accelerated in the forward shock, presumably driven by the AGN though with possible contribution by the starburst, that sweeps out the ISM generating the observed molecular outflow. Following FaucherGiguère \& Quataert (2012) and Nims et al. (2015), the predicted synchrotron emission associated with the $\mathrm{HVC}$ is given by $\nu L_{\nu} \sim 5.4 \times 10^{-6} \epsilon_{-2} L_{\mathrm{AGN}}\left(L_{\mathrm{kin}} / 0.027 L_{\mathrm{AGN}}\right)$, where $\epsilon=10^{-2} \epsilon_{-2}$ is the fraction of the forward shock energy flux that goes into relativistic electrons, and we have normalized the instantaneous mechanical power of the HVC in Mrk 231 to $2.7 \%$ of $L_{\mathrm{AGN}}$ (GA17, assuming that it is a factor of $\sim 4$ higher than the time-averaged value). Using $\epsilon_{-2}=1$ and $L_{\mathrm{AGN}}=8.6 \times 10^{45} \mathrm{erg} \mathrm{s}^{-1}$, the predicted monochromatic luminosity at $1.4 \mathrm{GHz}$ is $L_{1.4 \mathrm{GHz}} \sim 3 \times 10^{31} \mathrm{erg} \mathrm{s}^{-1} \mathrm{~Hz}^{-1}$, while 
the observed $1.4 \mathrm{GHz}$ luminosity from the inner disk of Mrk 231 is $5.3 \times 10^{30} \mathrm{erg} \mathrm{s}^{-1} \mathrm{~Hz}^{-1}$ (Carilli et al. 1998). Despite inverse-Compton losses may significantly decrease the predicted $1.4 \mathrm{GHz}$ luminosity, the excess of a factor $\sim 5$ relative to observations appears to indicate that there is enough energy flux in the forward shock associated with the molecular outflow to account for the observed disk-like radio emission (for the ionized phase, see Zakamska \& Greene 2014).

On the other hand, the CR proton luminosity (i.e. the energy flux associated with the CRs) is estimated as $\dot{E}_{\mathrm{CR}} \sim$ $2.7 \times 10^{-3}\left(\epsilon_{\mathrm{nt}} / 0.1\right) L_{\mathrm{AGN}}\left(L_{\mathrm{kin}} / 0.027 L_{\mathrm{AGN}}\right)$, where $\epsilon_{\mathrm{nt}} \sim 0.1$ is the assumed fraction of the forward energy flux that is converted into non-thermal energy flux of CR protons (Liu et al. 2017). The above expression yields $\dot{E}_{\mathrm{CR}} \sim 2 \times 10^{43} \mathrm{erg} \mathrm{s}^{-1}$, at least a factor of $\sim 2$ below our estimate from eq. (7). The discrepancy may be due to a higher value of $\epsilon_{\mathrm{nt}}$ for the lowenergy CRs causing the molecular ionization. In addition, and according to first-order Fermi acceleration, CRs gain energy by crossing and recrossing the shock front back and forth (e.g. Bell 2013), and the observed ionization rate may be caused by diffusion of these accelerating CRs into the outflowing molecular gas downstream. The process may be very efficient, as a given $\mathrm{CR}$ crosses the shock many times.

\section{CONCLUSIONS}

The main findings and conclusions of this study are:

1- Herschel/PACS spectroscopic observations of Mrk 231 show absorption in the $\mathrm{OH}^{+}$excited rotational lines at both systemic and blueshifted velocities up to $\sim-1000 \mathrm{~km} \mathrm{~s}^{-1}$. $\mathrm{OH}^{+}$is thereby an excellent tracer of the molecular outflow.

2- The $\mathrm{OH}^{+} 2_{2}-1_{1}$ and $2_{1}-1_{0}$ fine-structure lines at $\sim 150$ $\mu \mathrm{m}$ show blueshifted absorption wings very similar in shape to those observed in the $\mathrm{OH}$ doublets at 84 and $65 \mu \mathrm{m}$, indicating that both species share similar outflow regions.

3- Herschel/PACS observations of the excited $\mathrm{H}_{2} \mathrm{O}^{+}$and $\mathrm{H}_{3} \mathrm{O}^{+}$rotational lines only show absorption, if any, at systemic velocities, with no evidence for blueshifted wings. Most $\mathrm{H}_{3} \mathrm{O}^{+}$rotational lines are undetected.

4- Herschel/SPIRE observations show P-Cygni profiles in the ground-state $\mathrm{OH}^{+}$lines, with strong redshifted emission features. The ground-state $\mathrm{H}_{2} \mathrm{O}^{+} 1_{11}-0_{00} 1 / 2-1 / 2$ line shows strong emission above the continuum, but no hints of blueshifted absorption.

5- Radiative transfer models, similar to those previously reported for $\mathrm{OH}$ (GA17), have been applied to $\mathrm{OH}^{+}, \mathrm{H}_{2} \mathrm{O}^{+}$and $\mathrm{H}_{3} \mathrm{O}^{+}$. At systemic velocities, probing the nuclear torus of $\sim$ $100 \mathrm{pc}$ scale around the AGN, we find column density ratios of $\mathrm{OH} / \mathrm{OH}^{+} \sim 20, \mathrm{OH}^{+} / \mathrm{H}_{2} \mathrm{O}^{+} \sim 4-8$, and $\mathrm{OH}^{+} / \mathrm{H}_{3} \mathrm{O}^{+} \gtrsim 4$. For the outflowing gas, $\mathrm{OH} / \mathrm{OH}^{+} \sim 10$ and $\mathrm{OH}^{+} / \mathrm{H}_{2} \mathrm{O}^{+} \gtrsim 10$. The abundance of $\mathrm{OH}^{+}$relative to $\mathrm{H}$ nuclei is estimated to be high, $>10^{-7}$, in both components.

6- Chemical models are used to predict the ratios and abso- lute values of the abundances of $\mathrm{OH}, \mathrm{OH}^{+}, \mathrm{H}_{2} \mathrm{O}^{+}$, and $\mathrm{H}_{3} \mathrm{O}^{+}$, which are compared with the inferred values to estimate the ionization rate $\zeta$ of the molecular gas. We estimate $\zeta \sim 10^{-12}$ and $\sim 5 \times 10^{-13} \mathrm{~s}^{-1}$ in the nuclear torus and outflowing gas, respectively. The molecular fraction is expected to be low, $f_{\mathrm{H}_{2}}<0.5$.

7- The inferred high ionization rates, of order $10^{4}$ times those inferred in the Milky Way, are hard to explain by the relatively weak emission of Mrk 231 in X-rays, and therefore low-energy $(10-400 \mathrm{MeV})$ cosmic rays are proposed to play a primary role on the ionization of the molecular gas. Accounting for the energy loss as CRs travel through the (partially) molecular gas, we estimate a mass outflow rate and energy flux in low-energy $\mathrm{CRs}$ of $\dot{M}_{\mathrm{CR}} \sim 0.01 \mathrm{M}_{\odot} \mathrm{yr}^{-1}$ and $\dot{E}_{\mathrm{CR}} \sim 10^{44} \mathrm{erg} \mathrm{s}^{-1}$.

8- Diffusion of CRs downstream into the outflowing molecular gas, as they are accelerated through repeated crossings of the forward shock, may be an efficient way of accounting for the ionization rate of the outflowing gas. The forward shock associated with the molecular outflow in Mrk 231 also has enough energy to account for the disk-like synchrotron emission generated by relativistic electrons.

We thank Alexander Richings and Claude-André FaucherGiguère for useful discussions on models of energyconserving outflows, and Karen Yang and Ke Fang for commenting on the problem of CR propagation. E.GA is grateful for the warm hospitality of the Harvard-Smithsonian Center for Astrophysics, where most of the present study was carried out. PACS was developed by a consortium of institutes led by MPE (Germany) and including UVIE (Austria); KU Leuven, CSL, IMEC (Belgium); CEA, LAM (France); MPIA (Germany); INAFIFSI/OAA/OAP/OAT, LENS, SISSA (Italy); IAC (Spain). This development has been supported by the funding agencies BMVIT (Austria), ESA-PRODEX (Belgium), CEA/CNES (France), DLR (Germany), ASI/INAF (Italy), and CICYT/MCYT (Spain). E.GA is a Research Associate at the Harvard-Smithsonian Center for Astrophysics, and thanks the Spanish Ministerio de Economía y Competitividad for support under projects FIS2012-39162-C0601 and ESP2015-65597-C4-1-R. E.GA and H.A.S. thank NASA grant ADAP NNX15AE56G. Basic research in IR astronomy at NRL is funded by the US ONR; J.F. also acknowledges support from the NHSC. S.V. thanks NASA for partial support of this research via Research Support Agreement RSA 1427277, support from a Senior NPP Award from NASA, and his host institution, the Goddard Space Flight Center. This research has made use of NASA's Astrophysics Data System (ADS) and of GILDAS software (http://www.iram.fr/IRAMFR/GILDAS).

Facilities: Herschel/PACS and SPIRE 


\section{Software: GILDAS}

\section{REFERENCES}

Aalto, S., García-Burillo, S., Muller, S., et al. 2012, A\&A, 537, 44 Aalto, S., García-Burillo, S., Muller, S., Winters, J. M.,

González-Alfonso, E., van der Werf, P., Henkel, C., Costagliola, F., \& Neri, R. 2015, A\&A, 574, A85

Alatalo, K., Blitz, L., Young, L. M., et al. 2011, ApJ, 735, 88

Alatalo, K., Lacy, M., Lanz, L. 2015, ApJ, 798, 31

Bayet, E., Williams, D. A., Hartquist, T. W., \& Viti, S. 2011, MNRAS, 414, 1583

Bell, A. R. 2013, Astroparticle Physics, 43, 56

Bruderer, S., Doty, S. D., \& Benz, A. O. 2009, ApJS, 183, 179

Carilli, C. L., Wrobel, J. M., \& Ulvestad, J. S. 1998, ApJ, 115, 928

Cicone, C., Feruglio, C., Maiolino, R., et al. 2012, A\&A, 543, A99

Cicone, C., Maiolino, R., Sturm, E., et al. 2014, A\&A, 562, A21

Doty, S. D., van Dishoeck, E. F., van der Tak, F. F. S., \& Boonman, A. M. S. 2002, A\&A, 389, 446

Elitzur, M., \& de Jong, T. 1978, A\&A, 67, 323

Falstad, N., González-Alfonso, E., Aalto, S., van der Werf, P. P.,

Fischer, J., Veilleux, S., Meléndez, M., Farrah, D., \& Smith, H. A. 2015, A\&A, 580, A52

Faucher-Giguère, C.-A. \& Quataert, E. 2012, MNRAS, 425, 605

Feruglio, C., Maiolino, R., Piconcelli, E., et al. 2010, A\&A, 518, L155

Feruglio, C., Fiore, F., Carniani, S., et al. 2015, A\&A, 583, A99

Fischer, J., Sturm, E., González-Alfonso, et al. 2010, A\&A, 518, L41

García-Burillo, S., Combes, F., Usero, A., et al. 2015, A\&A, 580, A35

González-Alfonso, E., Smith, H. A., Ashby, et al. 2008, ApJ, 675, 303

González-Alfonso, E., Fischer, J., Isaak, K., et al. 2010, A\&A, 518, L43

González-Alfonso, E., Fischer, J., Graciá-Carpio, J., et al. 2012, A\&A, 541, A4

González-Alfonso, E., Fischer, J., Bruderer, S., et al. 2013, A\&A, 550, A25 (GA13)

González-Alfonso, E., Fischer, J., Graciá-Carpio, J., et al. 2014, A\&A, 561, A27 (GA14)

González-Alfonso, E., Fischer, Aalto, S., \& Falstad, N. 2014b, A\&A, 567, A91

González-Alfonso, E., Fischer, J., Sturm, E., et al. 2015, ApJ, 800, 69

González-Alfonso, E., Fischer, J., Spoon, H. W. W., et al. 2017, ApJ, 836, 11 (GA17)

Herbst, E., \& Klemperer, W. 1973, ApJ, 185, 505

Hollenbach, D., Kaufman, M. J., Neufeld, D., Wolfire, M., \& Goicoechea, J. R. 2012, ApJ, 754, 105
Indriolo, N., Fields, B. D., \& McCall, B., J. 2009, ApJ, 694, 257 Indriolo, N., Neufeld, D. A., Gerin, M., Geballe, T. R., Black, J. H., Menten, K. M., \& Goicoechea, J. R. 2012, ApJ, 758, 83 Indriolo, N., Neufeld, D. A., Gerin, M., et al. 2015, ApJ, 800, 40 Janssen, A. W., Christopher, N., Sturm, E., et al. 2016, ApJ, 822, 43 King, A. R., Zubovas, K., \& Power, C. 2011, MNRAS, 415, L6 Klöckner, H.-R., Baan, W. A., \& Garrett, M. A., 2003, Nature, 421, 821

Lindberg, J. E., Aalto, S., Muller, S., et al. 2016, A\&A, 587, A15 Lique, F., Bulut, N., \& Roncero, O. 2016, MNRAS, 461, 4477

Liu, R.-Y., Murase, K., Inoue, S., Ge, C., \& Wang, X.-Y. 2017, arXiv: 1712.10168

Maloney, P. R., Hollenbach, D, J., \& Tielens, A. G. G. M. 1996, ApJ, 466, 561

Markus, C. R., Hodges, J. N., Perry, A, J., Kocheril, G. S., Müller, H. S. P., \& McCall, B. J. 2016, ApJ, 817, 138

Mashian, N., Sturm, E., Sternberg, A., et al. 2015, ApJ, 802, 81

Meijerink, R., Spaans, M., Loenen, A. F., \& van der Werf, P. P. 2011, A\&A, 525, 119

Morganti, R., Veilleux, S., Oosterloo, T., Teng, S. H., \& Rupke, D. 2016, A\&A, 593, A30

Muller, S., Müller, H. S. P., Black, J. H., et al. 2016, A\&A, 595, A128

Müller, H. S. P., Thorwirth, S., Roth, D. A., \& Winnewisser, G. 2001, A\&A, 370, L49

Müller, H. S. P., Schlöder, F., Stutzki, J., \& Winnewisser, G. 2005, J. Mol. Struct. 742, 215

Neufeld, D. A., et al. 2010, A\&A, 521, L10

Nims, J., Quataert, E., \& Faucher-Giguère, C.-A. 2015, MNRAS, 447, 3612

Padovani, M., Galli, D., \& Glassgold, A. E. 2009, A\&A, 501, 619

Padovani, M., \& Galli, D. 2011, A\&A, 530, A109

Papadopoulos, P. P., Thi, W.-F., Miniati, F., \& Viti, S. 2011, MNRAS, 414, 1705

Papadopoulos, P. P.; Zhang, Z.-Y., Xilouris, E. M., et al. 2014, ApJ, 788, 153

Pickett, H. M., Poynter, R. L., Cohen, E. A., Delitsky, M. L., Pearson, J. C., \& Müller, H. S. P. 1998, JQSRT, 60, 883

Pilbratt, G. L., Riedinger, J. R., Passvogel, T., et al. 2010, A\&A, 518, L1

Poglitsch, A., Waelkens, C., Geis, N., et al. 2010, A\&A, 518, L2

Rangwala, N., Maloney, P. R., Glenn, J., et al. 2011, ApJ, 743, 94

Reynolds, C., Punsly, B., Miniutti, G., O’Dea, C. P., \& Hurley-Walker, N. 2017, ApJ, in press (arXiv:1701.03190)

Richings, A. J., \& Faucher-Giguère, C.-A. 2018, MNRAS, 474, 3673 
Richings, A. J., \& Faucher-Giguère, C.-A. 2017, MNRAS (arXiv:1710.09433)

Rosenberg, M. J. F., van der Werf, P. P., Aalto, S., et al. 2015, ApJ, 801,72

Sakamoto, K., Aalto, S., Wilner, D. J., et al. 2009, ApJ, 700, L104

Spitzer, L. Jr., \& Tomasko, M. G. 1968, ApJ, 152, 971

Spoon, H. W. W., Farrah, D., Lebouteiller, V., et al. 2013, ApJ, 775, 127

Stäuber, P., Doty, S. D., van Dishoeck, E. F., \& Benz, A. O. 2005, A\&A, 440, 949

Stone, M., Veilleux, S., Meléndez, M., Sturm, E., Graciá-Carpio, J., \& González-Alfonso, E., 2016, ApJ, 826, 111

Stone, M., Veilleux, S., González-Alfonso, E., Spoon, H. W. W., \& Sturm, E. 2017, ApJ

Sturm, E., González-Alfonso, E., Veilleux, S., et al. 2011, ApJ, 733, L16 (S11)

Taylor, G. B., Silver, C. S., Ulvestad, J. S., \& Carilli, C. L. 1999, ApJ, 519, 185

Teng, S. H., Brandt, W. N., Harrison, F. A., et al. 2014, ApJ, 785, 19

Tombesi, F., Meléndez, M., Veilleux, S., Reeves, J. N., González-Alfonso, E., \& Reynolds, C. S. 2015, Nature, 519, 436
Ulvestad, J. S., Wrobel, J. M., \& Carilli, C. L. 1999, ApJ, 516, 127 van der Tak, F. F. S., Nagy, Z., Ossenkopf, V., Makai, Z., Black, J. H., Faure, A., Gerin, M., \& Bergin, E. A. 2013, A\&A, 560, A95 van der Tak, F. F. S., Weiß, A., Liu, L., \& Güsten, R. 2016, A\&A, 593, A43

van der Werf, P. P., Isaak, K. G., Meijerink, R., et al. 2010, A\&A, 518, L42

van Dishoeck, E. F., Jansen, D. J., Schilke, P., \& Phillips, T. G. 1993, ApJ, 416, L83

Veilleux, S., Meléndez, M.; Sturm, E., et al. 2013a, ApJ, 776, 27

Veilleux, S., Trippe, M., Hamann, F., et al. 2013b, ApJ, 764, 15

Veilleux, S., Meléndez, M., Tripp, T. M., Hamann, F., \& Rupke, D. S. N. 2016, ApJ, 825, 42

Veilleux, S., Bolatto, A., Tombesi, F., Meléndez, M., Sturm, E., González-Alfonso, E., Fischer, J., \& Rupke, D. S. N. 2017, ApJ, 843,18

Woodall, J., Agúndez, M., Markwick-Kemper, A. J., \& Millar, T. J. 2007, A\&A 466, 1197

Zakamska, N. L. \& Greene, J. E. 2014, MNRAS, 442, 784

Zubovas, K., \& King, A. 2012, ApJ, 745, L34

Zubovas, K., \& King, A. 2014, MNRAS, 439, 400 